\title{
Cost-effective design for a neutrino factory
}

\author{
J. S. Berg, ${ }^{1}$ S. A. Bogacz, ${ }^{2}$ S. Caspi,${ }^{3}$ J. Cobb,${ }^{4}$ R. C. Fernow, ${ }^{1}$ J. C. Gallardo, ${ }^{1}$ S. Kahn,,${ }^{1}$ H. Kirk,,${ }^{1}$ D. Neuffer, ${ }^{5}$ R. Palmer, ${ }^{1}$ \\ K. Paul, ${ }^{6} \mathrm{H}$. Witte, ${ }^{4}$ and M. Zisman ${ }^{3}$ \\ ${ }^{1}$ Brookhaven National Laboratory, Upton, New York 11973, USA \\ ${ }^{2}$ Jefferson Laboratory, 12000 Jefferson Avenue, Newport News, Virginia 23606, USA \\ ${ }^{3}$ Lawrence Berkeley National Laboratory, Berkeley, California 94720, USA \\ ${ }^{4}$ Oxford University, Oxford OX1 3RH, United Kingdom \\ ${ }^{5}$ Fermi National Accelerator Laboratory, Batavia, Illinois 60510, USA \\ ${ }^{6}$ Muons Inc., Batavia, Illinois 60510, USA
}

(Received 14 November 2005; published 24 January 2006)

\begin{abstract}
There have been active efforts in the U.S., Europe, and Japan on the design of a neutrino factory. This type of facility produces intense beams of neutrinos from the decay of muons in a high-energy storage ring. In the U.S., a second detailed feasibility study (FS2) for a neutrino factory was completed in 2001. Since that report was published, new ideas in bunching, cooling, and acceleration of muon beams have been developed. We have incorporated these ideas into a new facility design, which we designate as study 2B (ST2B), that should lead to significant cost savings over the FS2 design.
\end{abstract}

DOI: 10.1103/PhysRevSTAB.9.011001

PACS numbers: 29.20.Dh, 41.75.Lx, 14.60.Ef

\section{INTRODUCTION}

A neutrino factory [1-4] facility offers an exciting option for the long-term neutrino physics program. In the U.S. there has been a significant investment in developing the concepts and technologies required for such an accelerator complex. New accelerator technologies offer the possibility of building, in the not-too-distant future, an accelerator complex to produce more than $10^{20}$ muons per year [3]. It has been proposed to build a neutrino factory by accelerating the muons from this intense source to energies of tens of $\mathrm{GeV}$, injecting them into a storage ring having long straight sections, and exploiting the intense neutrino beams that are produced by muons decaying in the straight sections. The decays

$$
\mu^{-} \rightarrow e^{-} \nu_{\mu} \bar{\nu}_{e}, \quad \mu^{+} \rightarrow e^{+} \bar{\nu}_{\mu} \nu_{e}
$$

yield neutrinos that are directed along the line of the straight sections. This allows them to be observed at near and far detectors, and offers exciting possibilities to pursue the study of neutrino oscillations and neutrino interactions with exquisite precision.

A neutrino factory requires an intense multi-GeV proton source capable of producing a primary proton beam with a beam power of 1-4 MW or more on target. This is the same proton source required in the medium term for neutrino superbeams; hence, there is a natural evolution from Superbeam experiments to neutrino factory experiments.

The physics case for a neutrino factory will depend upon results from the next round of planned neutrino oscillation experiments [5]. If the unknown mixing angle $\theta_{13}$ is small, such that $\sin ^{2} 2 \theta_{13}<O\left(10^{-2}\right)$, or if there is a surprise and three-flavor mixing does not completely describe the observed phenomenology, then answers to some or all of the most important neutrino oscillation questions will require a neutrino factory. If $\sin ^{2} 2 \theta_{13}$ is large, just below the present upper limit, and if there are no experimental surprises, the physics case for a neutrino factory will depend on the values of the oscillation parameters, the achievable sensitivity that will be demonstrated by the first generation of $\nu_{e}$ appearance experiments, and the nature of the second generation of basic physics questions that will emerge from the first round of results. In either case (large or small $\theta_{13}$ ), in about a decade the neutrino community may need to insert a neutrino factory into the global neutrino plan. The option to do this in the next ten years will depend upon the accelerator research and development that is done during the intervening period.

In the U.S., the Neutrino Factory and Muon Collider Collaboration (referred to herein as the NFMCC [6]) is a collaboration of 130 scientists and engineers engaged in carrying out the accelerator research and development that is needed before a neutrino factory could be inserted into the global plan. Much technical progress has been made over the last few years, and several of the required key accelerator experiments are now approved. In addition to the U.S. effort, there are active neutrino factory research and development groups in Europe [7,8] and Japan [9], and much of the research and development is performed and organized as an international endeavor. Thus, because a neutrino factory is potentially the key facility for the longterm neutrino program, neutrino factory research and development is an important part of the present global neutrino program. The key research and development experiments are seeking funding now, and will need to be supported if Neutrino Factories are to be an option for the future.

In this article we describe an updated neutrino factory design that demonstrates significant progress toward performance improvements and cost reduction for this ambi- 


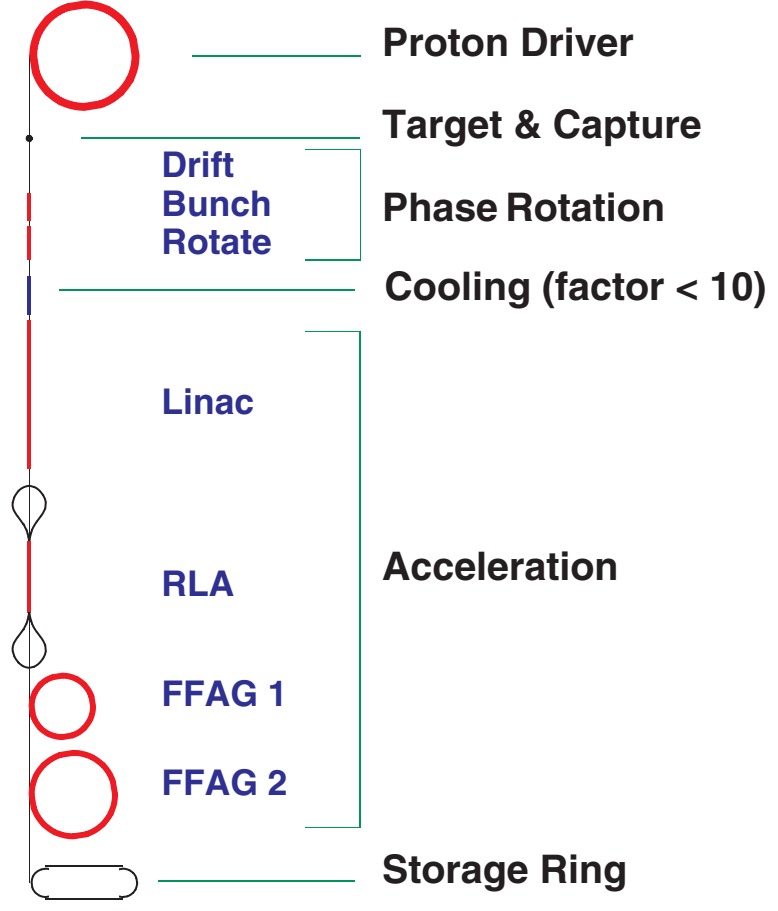

FIG. 1. (Color) Schematic of a neutrino factory facility.

tious facility. The paper is organized as follows: . Section II describes the neutrino factory design concept. The design of the front end of the facility is described in Sec. III and the accelerator chain is described in Sec. IV. In Sec. V we discuss the storage ring and the overall performance. Required research and development is described in Sec. VI. In Sec. VII we discuss the assumptions used to make the cost estimate for a neutrino factory and finally, we conclude with a summary in Sec. VIII.

Much of the work described in this paper was performed as part of the year-long study of the physics of neutrinos, organized by the American Physical Society [5].

\section{MACHINE CONCEPT}

In this section we describe the basic machine concepts that are used to create a neutrino factory facility $[1,5,10]$; a schematic of the whole facility is shown in Fig. 1. The facility is a quaternary beam machine; that is, a primary proton beam is used to create first a secondary pion beam and subsequently, a tertiary muon beam that decays and eventually provides the neutrino flux for the detector. For a neutrino factory the primary beam is a high intensity proton beam of moderate energy (beams of $2-50 \mathrm{GeV}$ have been considered by various groups) that impinges on a target, typically a high- $Z$ material (e.g., $\mathrm{Hg}$ ). The collisions between the proton beam and the target nuclei produce a secondary pion beam that quickly decays $(26.0 \mathrm{~ns})$ into a longer-lived $(2.2 \mu \mathrm{s})$ muon beam. The remainder of the neutrino factory is used to condition the muon beam (see Sec. III), accelerate it rapidly to the desired final energy of a few tens of GeV (see Sec. IV), and store it in a decay ring having a long straight section oriented such that decay neutrinos produced there will hit a detector located thousands of kilometers from the source.

Two feasibility studies $[1,10]$ have demonstrated technical feasibility (provided the challenging component specifications are met), established a cost baseline, and established the expected range of physics performance. Our present concept of a neutrino factory is based in part on the most recent feasibility study (study-II, referred to herein as FS2) [1] that was carried out jointly by BNL and the U.S. NFMCC. It is worth noting that the neutrino factory design we envision could fit comfortably on the site of an existing laboratory, such as BNL or FNAL. Figure 1 shows a schematic of the facility. A summary of parameters is given in Table I.

The main ingredients of a neutrino factory include:

(i) Proton Driver - 1-4 MW (1 MW in this article, but possibly upgradeable to $4 \mathrm{MW}$ ) of protons on target from, for example, an upgraded alternating gradient synchrotron (AGS); a new booster at Fermilab (or elsewhere) would perform equivalently.

(ii) Target and capture.-A high-power target is immersed in a $20 \mathrm{~T}$ superconducting solenoidal field to capture pions produced in proton-nucleus inter-

TABLE I. Summary of the main parameters.

\begin{tabular}{lc}
\hline \hline Proton energy $(\mathrm{GeV})$ & 24 \\
Driver cycle rate $(\mathrm{Hz})$ & 2.5 \\
Bunches per spill & 6 \\
Average bunch rate $(\mathrm{Hz})$ & $2.5 \times 6=15$ \\
Protons per bunch $\left(10^{12}\right)$ & 1.6 \\
Bunch length, rms (ns) & 3 \\
Proton power (MW) & 1 \\
Final muon energy $(\mathrm{GeV})$ & 20 \\
Muons of each sign per proton after cooling & 0.17 \\
Muons of each sign per proton after acceleration & 0.11 \\
Muons of both signs per $10^{7}$ sec decaying toward detector & $2 \times 10^{20}$ \\
\hline \hline
\end{tabular}


actions. The high magnetic field at the target is smoothly tapered down to a much lower value, $1.75 \mathrm{~T}$, which is then maintained through the bunching and phase rotation sections of the neutrino factory.

(iii) Bunching and phase rotation.-We first accomplish the bunching with rf cavities of modest gradient, whose frequencies change as we proceed down the beam line. After bunching the beam, another set of rf cavities, with higher gradients and again having decreasing frequencies as we proceed down the beam line, is used to rotate the beam in longitudinal phase space to reduce its energy spread.

(iv) Cooling. - A solenoidal focusing channel, with high-gradient $201.25 \mathrm{MHz}$ rf cavities and $\mathrm{LiH}$ absorbers, cools the transverse normalized rms emittance from $17 \mathrm{~mm} \cdot \operatorname{rad}$ to about $7 \mathrm{~mm} \cdot \operatorname{rad}$. This takes place at a central muon momentum of $220 \mathrm{MeV} / c$.

(v) Acceleration. - A superconducting linac with solenoidal focusing is used to raise the muon beam energy to $1.5 \mathrm{GeV}$, followed by a recirculating linear accelerator (RLA), arranged in a dog-bone geometry, to provide a $5 \mathrm{GeV}$ muon beam. Thereafter, a pair of cascaded fixed-field, alternating-gradient (FFAG) rings, with a triplet lattice of combined-function magnets, is used to reach $20 \mathrm{GeV}$. Additional FFAG stages could be added to reach a higher beam energy, if the physics requires this.

(vi) Storage ring. - We employ a compact racetrackshaped superconducting storage ring in which $\approx$ $35 \%$ of the stored muons decay while traveling toward detectors located nearby, and some $3000 \mathrm{~km}$ from the ring. Muons survive for roughly 500 turns.

In the remainder of this paper we describe in detail the new design of the neutrino factory front end for performing the required beam manipulations prior to acceleration and describe our new ideas for accelerating the muon beam using FFAGs.

\section{FRONT-END DESIGN}

Some front-end parameters are given in Table II. The front end of the neutrino factory (the part of the facility between the target and the first linear accelerator) represented a large fraction, about $40 \%$, of the total facility costs in FS2 [1]. However, several recent developments have led to a new design for the front end that has a crucial performance advantage and is also significantly less expensive. The new concepts are:

(i) A new approach to bunching and phase rotation using the concept of adiabatic of bunching [11-14]
TABLE II. Summary of the front-end parameters.

\begin{tabular}{lc}
\hline \hline Solenoid capture magnetic field $(\mathrm{T})$ & 20 \\
Length of taper $(\mathrm{m})$ & 12 \\
Solenoid field at end of taper $(\mathrm{T})$ & 1.75 \\
Length of drift $(\mathrm{m})$ & 99 \\
Solenoid field in drift $(\mathrm{T})$ & 1.75 \\
Length of buncher $(\mathrm{m})$ & 50 \\
Solenoid field in buncher $(\mathrm{T})$ & 1.75 \\
Maximum rf gradient in buncher $(\mathrm{MV} / \mathrm{m})$ & 10 \\
Length of phase rotator $(\mathrm{m})$ & 54 \\
Solenoid field in phase rotator $(\mathrm{T})$ & 1.75 \\
RF gradient in phase rotator $(\mathrm{MV} / \mathrm{m})$ & 12.5 \\
Length of cooler $(\mathrm{m})$ & 80 \\
Maximum solenoid field in cooler $(\mathrm{T})$ & 3 \\
RF gradient in cooler $(\mathrm{MV} / \mathrm{m})$ & 15.25 \\
\hline \hline
\end{tabular}

eliminates the very expensive induction linacs used in FS2.

(ii) For a moderate cost, the transverse acceptance of the accelerator chain is doubled from its FS2 value.

(iii) The increased accelerator acceptance diminishes the demands on the transverse ionization cooling and allows the design of a simplified cooling section with fewer components and reduced magnetic field strength.

We denote as Study 2B (ST2B) the simulations that have been made of the performance of this new front end, together with the new scheme for acceleration. The Monte Carlo simulations were performed with the code ICOOL [15]. The concept of the adiabatic buncher is compared with the system used in FS2 in Fig. 2. The longitudinal phase space after the target is the same in both cases. Initially, there is a small spread in time, but a very large spread in energy. The target is followed by a drift space in both cases, where a strong correlation develops between time and energy. Figure 3 shows the longitudinal phase space after the long drift. In FS2 the energy spread in the correlated beam was first flattened (phase rotated) using a series of induction linacs. The induction linacs did an excellent job, reducing the final rms energy spread to $4.4 \%$, but were expensive. The beam was then sent through a series of rf cavities for bunching, which increased the energy spread to $\approx 8 \%$.

In the new scheme, the correlated beam is first adiabatically bunched using a series of rf cavities with decreasing frequencies and increasing gradients in such a way that the bunch centers remain at the rf zero crossings, even as their spacing increases because of their differing energies and velocities. The buncher was designed by picking two reference particles with different momenta (e.g. 155 and $280 \mathrm{MeV} / c$ ) in the phase space distribution shown in Fig. 3. These two reference particles were defined to be a large integer number (e.g. 18) of rf wavelengths apart. Because of their velocity difference the reference particles became more separated in time as they proceeded to fixed 
Study2 (FS2) with Induction Linacs

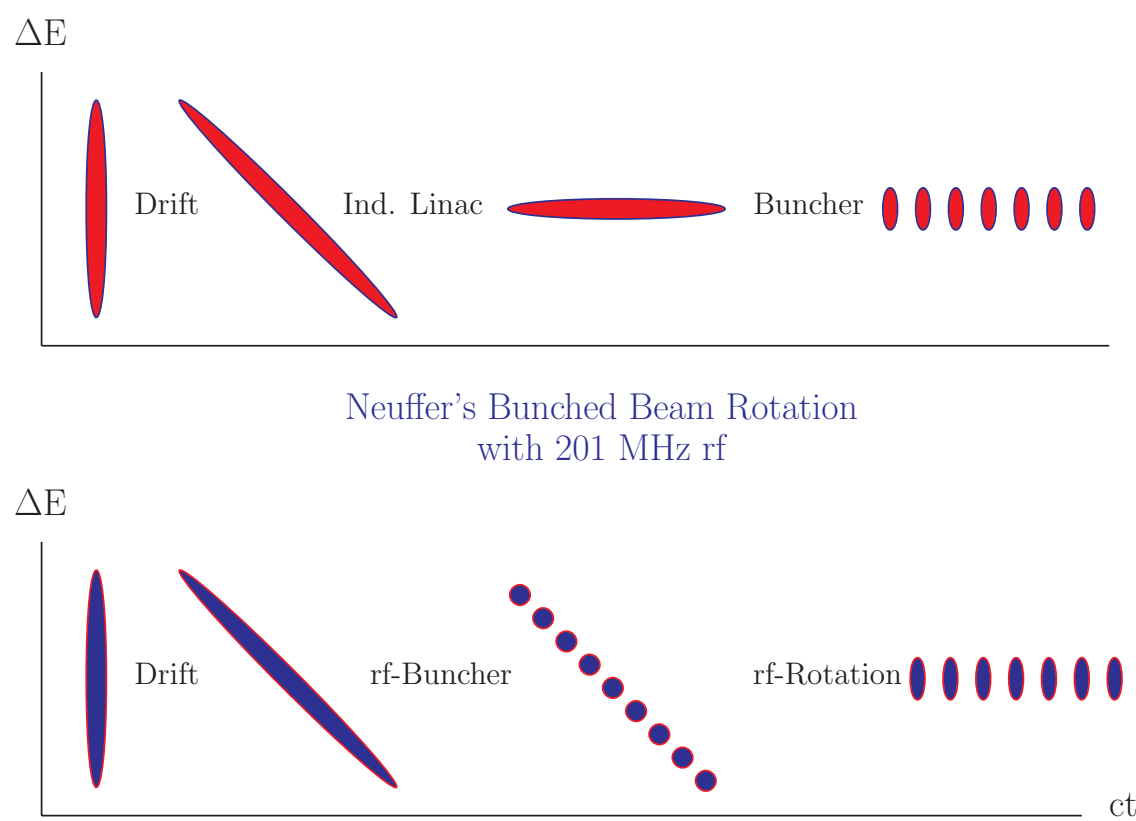

FIG. 2. (Color) Comparison of the buncher concept used here (bottom) with the bunching system used in FS2 (top).

locations in the buncher region. Since by construction the time difference always corresponds to a fixed number of wavelengths, the rf periods became longer and the corresponding $\mathrm{rf}$ frequencies fell as a function of distance along the channel.

The beam is then phase rotated with a second string of $\mathrm{rf}$ cavities with decreasing frequencies and constant gradient. In this case the frequencies are chosen with a slightly different criterion than that in the bunching. They are chosen so that the high-energy bunch centers see a decelerating rf field, while the low energy particles see an accelerating field. The final rms energy spread in the new design is $10.5 \%$. This spread is acceptable for the new cooling channel. The overall layout of the new front-end design is shown schematically in Fig. 4.

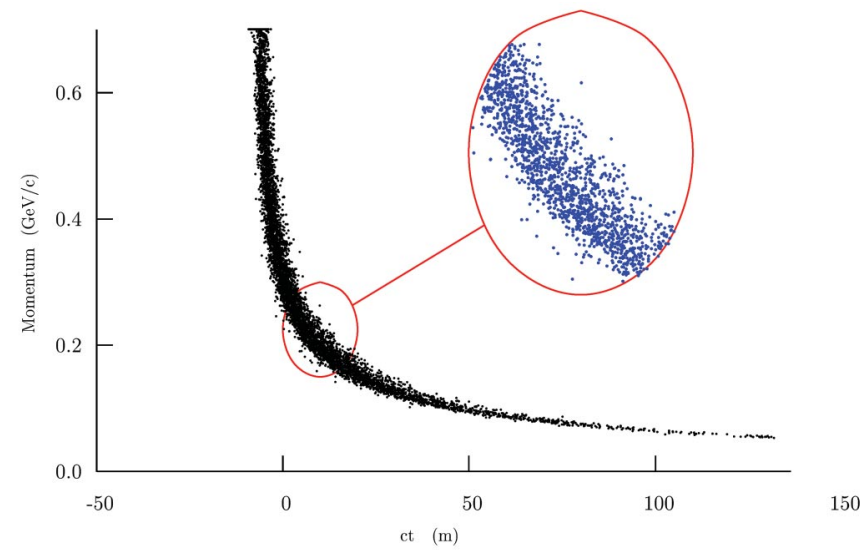

FIG. 3. (Color) Longitudinal phase space after the drift section.
The first $12 \mathrm{~m}$ is used to capture pions produced in the target. The field here drops adiabatically from 20 T over the target to $1.75 \mathrm{~T}$. At the same time, the radius of the beam pipe increases from $7.5 \mathrm{~cm}$ at the target up to $25 \mathrm{~cm}$. Next comes $99 \mathrm{~m}$ for the pions to decay into muons and for the energy-time correlation to develop. The adiabatic bunching occupies the next $50 \mathrm{~m}$ and the phase rotation and matching take place in $54 \mathrm{~m}$ following that. Last, the channel has $80 \mathrm{~m}$ of ionization cooling. The total length of the new front end is $295 \mathrm{~m}$. The longitudinal component of the magnetic field on-axis is shown for the full front end in Fig. 5. The field falls very rapidly in the collection region to a value of $1.75 \mathrm{~T}$. It keeps this value with very little ripple over the decay, buncher and rotator regions. After a short matching section, the $1.75 \mathrm{~T}$ field is changed to the alternating field used in the cooling section.

\section{A. Target and decay region}

The beam distributions used in the simulations were generated using MARS [16]. The distribution was calculated for a $24 \mathrm{GeV}$ proton beam interacting with a $\mathrm{Hg}$ jet [17].

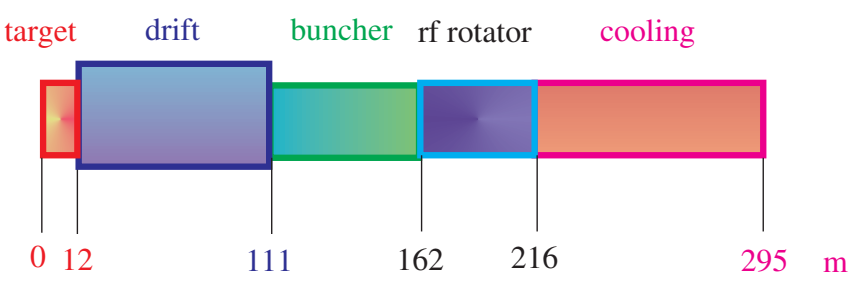

FIG. 4. (Color) Overall layout of the front end. 


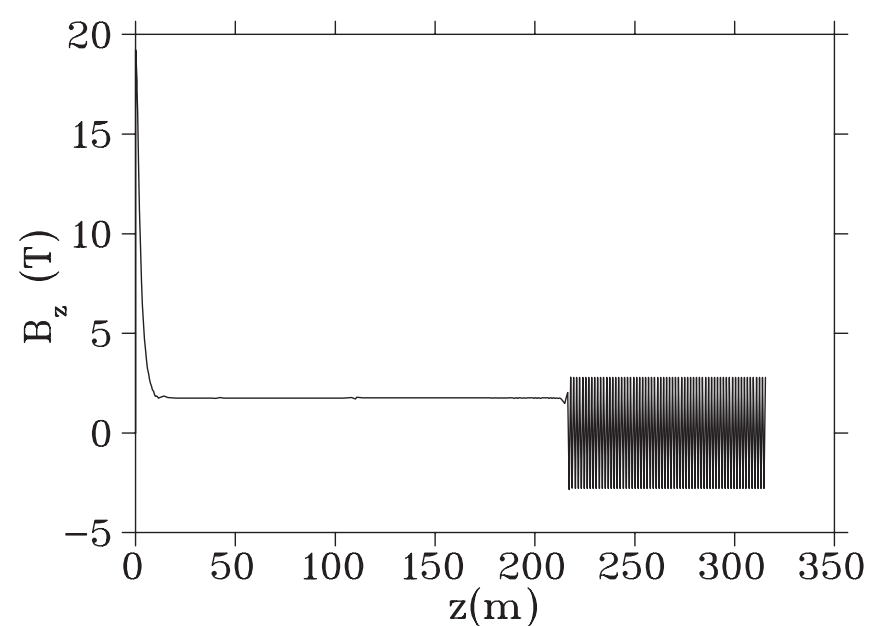

FIG. 5. Longitudinal field component $B_{z}$ on-axis along the ST2B front end.

The jet was incident at an angle of $100 \mathrm{mrad}$ to the solenoid axis, whereas the beam was incident at an angle of $67 \mathrm{mrad}$ to the solenoid axis. An independent study showed that the resulting $33 \mathrm{mrad}$ crossing angle gives near-peak acceptance for the produced pions. An examination of the distribution of particles that were propagated to the end of the capture region showed that they have a peak initial longitudinal momentum of $\approx 300 \mathrm{MeV} / c$ with a long highenergy tail, and a peak initial transverse momentum $\approx$ $180 \mathrm{MeV} / c$.

We used an improved axial field profile in the capture region that increased the final number of muons per proton in the accelerator acceptance by $\approx 10 \%$. The new axial field profile (marked ST2B) is compared in Fig. 6 with the profile used in FS2. Figure 7 shows the actual coil configuration in the collection region. The end of the $60 \mathrm{~cm}$ long

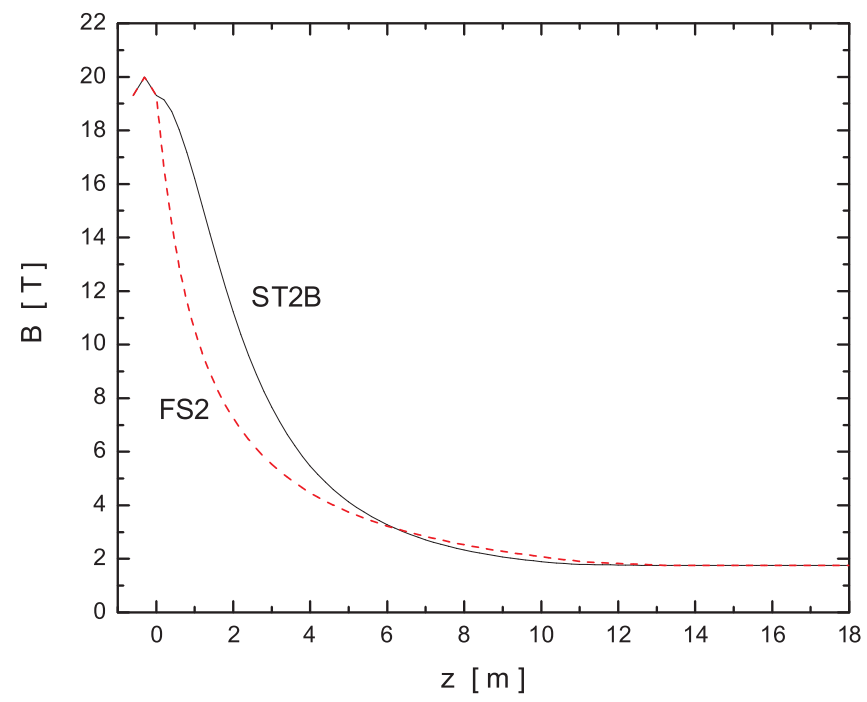

FIG. 6. (Color) Comparison of the capture region magnetic field used in the present simulation (ST2B) with that used in FS2.

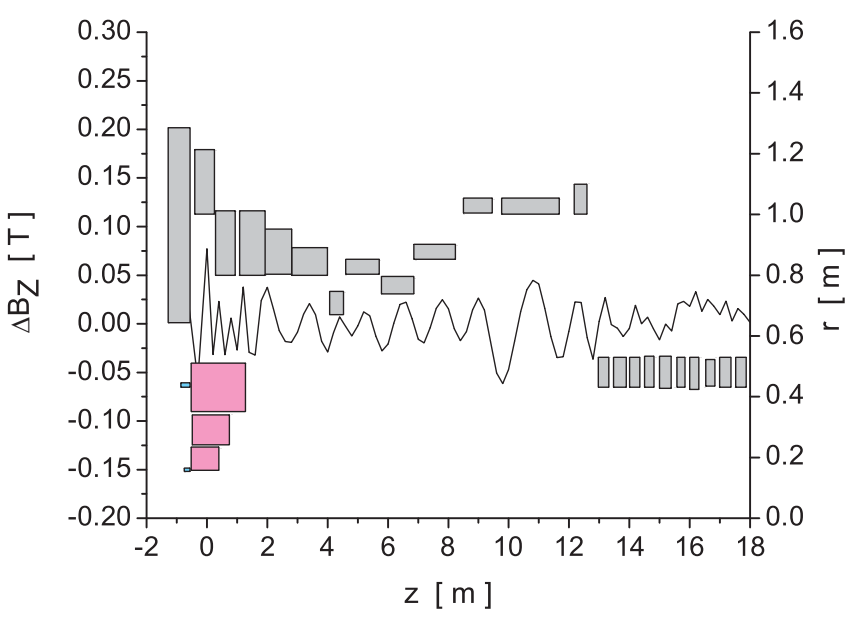

FIG. 7. (Color) Actual coil configuration in the collection region. The left axis shows the error field on-axis compared with the optimal capture field profile, denoted ST2B in Fig. 6.

target region is defined as $z=0$. The three small-radius coils near $z=0$ are $\mathrm{Cu}$ coils, while the others are superconducting. The left axis shows the error field on-axis compared with the desired field profile. We see that the maximum error field is $\approx 0.07 \mathrm{~T}$.

Figure 8 shows a MARS calculation of the absorbed radiation dose in the collection region. The peak energy deposition dose in the superconducting coils, as illustrated in Fig. 8 , is $\approx 0.5 \times 10^{-8} \mathrm{GeV} / \mathrm{g}$ per proton on target.

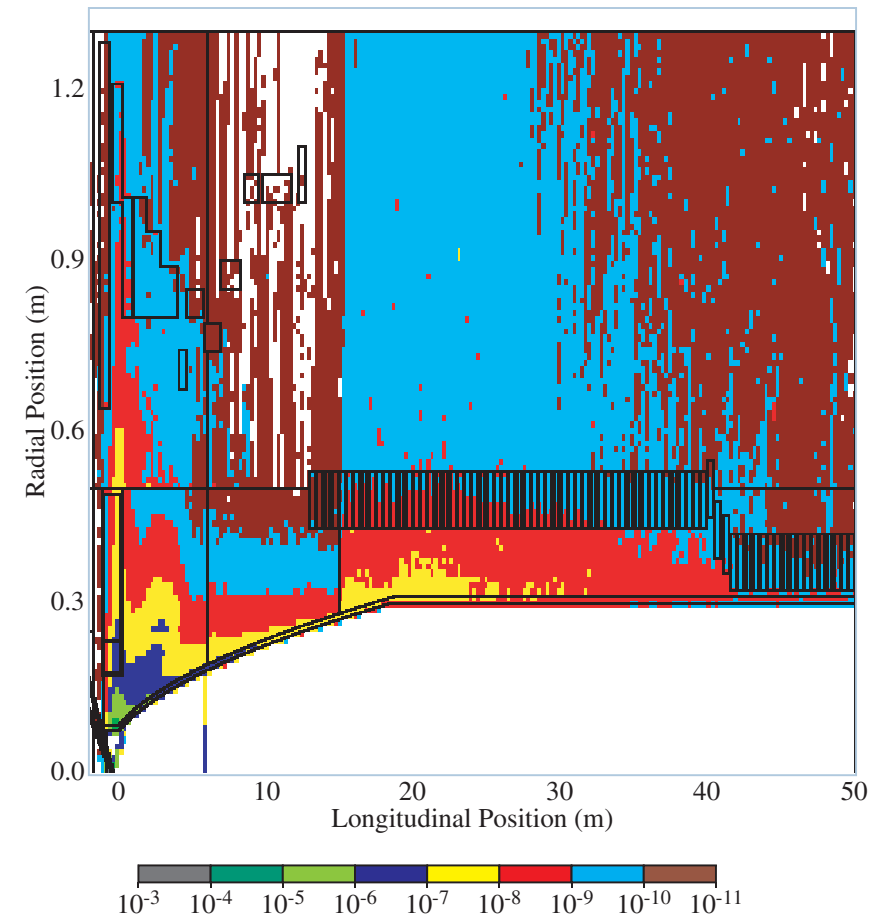

FIG. 8. (Color) MARS calculation of the absorbed annual radiation dose in the collection region for a $1 \mathrm{MW}$ proton beam. The scale shown is in units of $\mathrm{GeV} / \mathrm{g}$ per proton on target. 
This dose is $\approx 1 \mathrm{MGy} / \mathrm{yr}$ for a $1 \mathrm{MW}$ beam running for a Snowmass year of $1 \times 10^{7} \mathrm{~s}$. Assuming a lifetime dose for the insulation of $100 \mathrm{MGy}$, there should be no problem with radiation damage in the coils, even at a $4 \mathrm{MW}$ intensity level.

\section{B. Bunching and phase rotation region}

A cell of the buncher lattice is shown schematically in Fig. 9. Most of the cell length is occupied by the $50 \mathrm{~cm}$ long rf cavities. The cavity iris is covered with a Be window. The window thicknesses varied from 200 to $395 \mu \mathrm{m}$, depending on the cavity gradient. The limiting radial aperture in the cell is determined by the $25 \mathrm{~cm}$ radius of the window. The $50 \mathrm{~cm}$ long solenoids were placed outside the rf cavity with periodicity of $75 \mathrm{~cm}$, in order to decrease the magnetic field ripple on the axis and minimize beam losses from momentum stop bands. The buncher section contains 27 cavities with 13 discrete frequencies and gradients varying from $5-10 \mathrm{MV} / \mathrm{m}$.

The frequencies decrease from 333 to $234 \mathrm{MHz}$ in the buncher region. The cavities are not equally spaced. Fewer cavities are used at the beginning where the required gradients are small. Figure 10 shows the correlated longitudinal phase space and the bunching produced by the buncher.

The phase rotator cell is very similar to the buncher cell. The major difference is the use of tapered Be windows on the cavities because of the higher rf gradient. The tapered window had a thickness of $750 \mu \mathrm{m}$ from the axis out to a radius of $20 \mathrm{~cm}$ and a thickness of $1.5 \mathrm{~mm}$ from 20 to $25 \mathrm{~cm}$. There are 72 cavities in the rotator region, with 15 different frequencies. The frequencies decrease from 232 to $201 \mathrm{MHz}$ in this part of the front end. All cavities have a gradient of $12.5 \mathrm{MV} / \mathrm{m}$. Figure 11 shows the longitudinal phase space after the phase rotator. The rms energy spread in the beam is reduced to $27 \mathrm{MeV}$. To study the effect of a shorter phase rotator, we also considered an example having only a $26 \mathrm{~m}$ phase rotation section [18]. This alternative design would be significantly less expensive, since it is not only shorter but requires about $200 \mathrm{MV}$ less high-gradient rf voltage. Initial evaluations indicate a small decrease in captured muons $(\approx 10 \%)$.

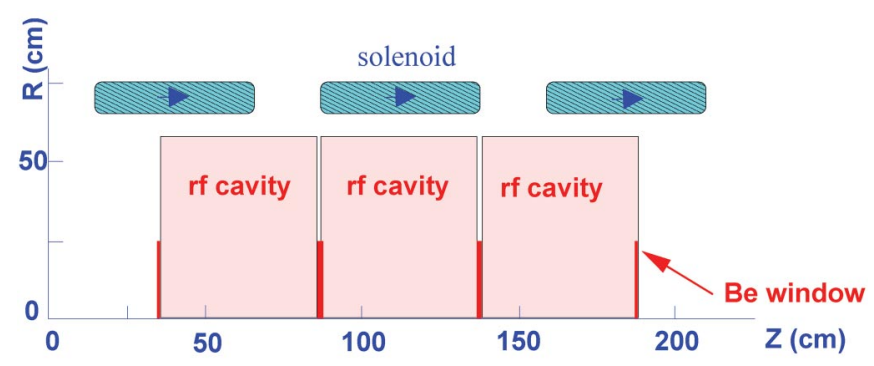

FIG. 9. (Color) Schematic of a cell at the beginning of the phase rotator section.

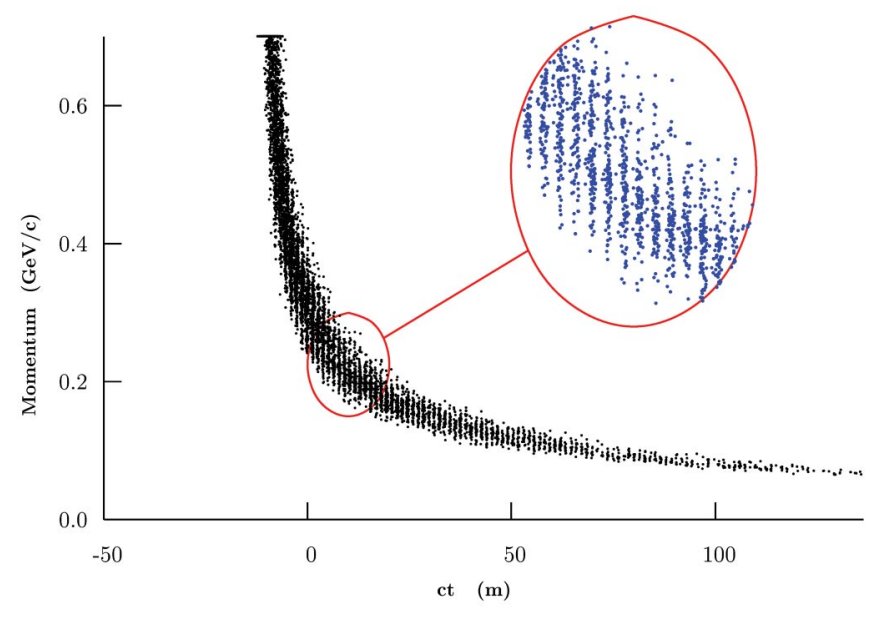

FIG. 10. (Color) Longitudinal phase space of one sign muons after the buncher section.

\section{Cooling region}

The cooling channel was designed to have a transverse beta function that is relatively constant with position and has a magnitude of about $80 \mathrm{~cm}$. One cell of the channel is shown in Fig. 12. Most of the $150 \mathrm{~cm}$ magnetic cell length is taken up by two $50 \mathrm{~cm}$ long rf cavities. The cavities have a frequency of $201.25 \mathrm{MHz}$ and a gradient of $15.25 \mathrm{MV} / \mathrm{m}$. A novel aspect of this design comes from using the windows on the rf cavity as the cooling absorbers. This is possible because the near-constant $\beta$ function eliminates the need to place the absorbers at a low- $\beta$ point to prevent emittance heating. The window consists of a $1 \mathrm{~cm}$ thickness of $\mathrm{LiH}$ with a $300 \mu \mathrm{m}$ thick layer of Be on the side facing the rf cavity field and a $25 \mu \mathrm{m}$ thick layer of $\mathrm{Be}$ on the opposite side. The Be will, in turn, have a thin coating of TiN to prevent multipactoring [19]. A $1 \mathrm{~cm}$ space might be introduced between the Be rf window and $\mathrm{LiH}$ absorber with flowing $\mathrm{He}$ gas to cool both. The alter-

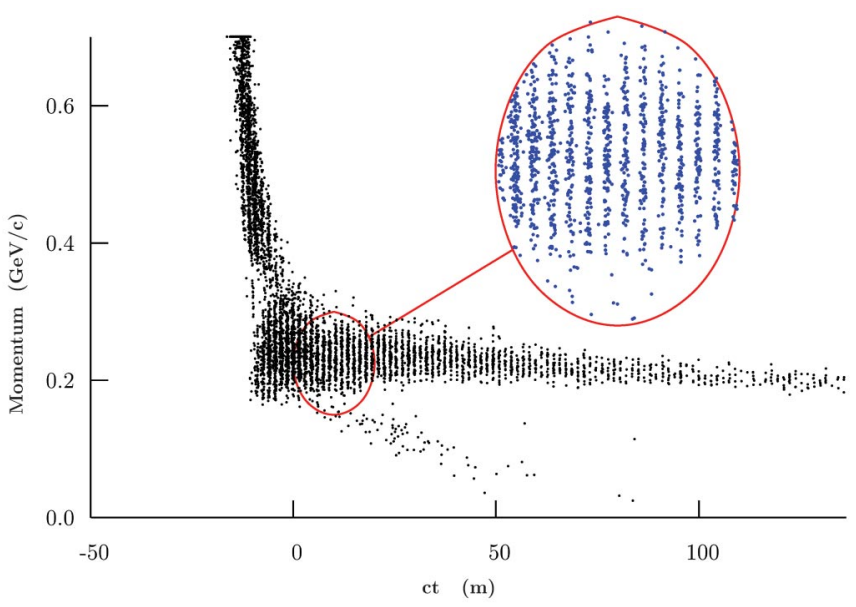

FIG. 11. (Color) Longitudinal phase space after the phase rotation section. 


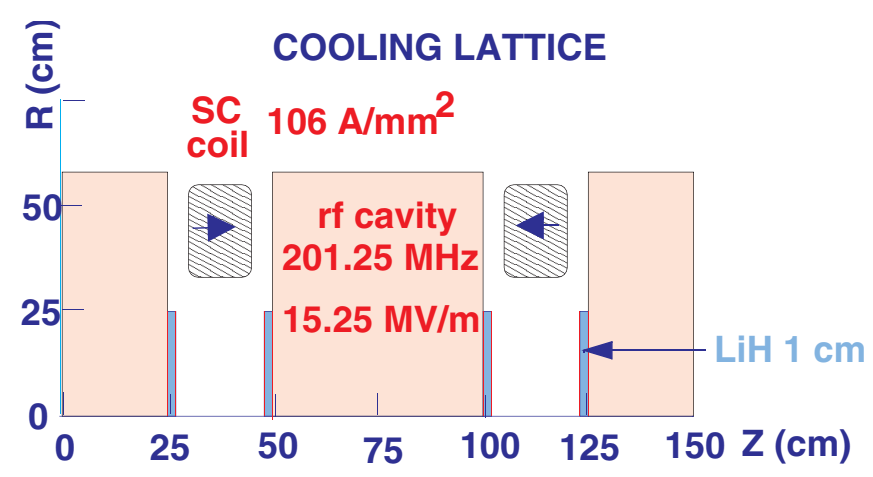

FIG. 12. (Color) Schematic of one cell of the cooling section.

nating $2.8 \mathrm{~T}$ solenoidal field is produced with one solenoid per half cell, located between the rf cavities.

Figure 13 shows the longitudinal phase space of one muon sign at the end of the cooling section. The reduction in normalized transverse emittance $\left(\epsilon_{T}\right)$ along the cooling channel is shown in the left plot of Fig. 14 and the right plot shows the normalized longitudinal emittance $\left(\epsilon_{L}\right)$. The channel produces a final value of $\epsilon_{T}=7.4 \mathrm{~mm} \mathrm{rad}$, which is more than a factor of 2 reduction from the initial value. The equilibrium value for a $\mathrm{LiH}$ absorber with an $80 \mathrm{~cm} \beta$ function is about $5.5 \mathrm{~mm}$ rad. Figure 15 shows the muons per incident proton on target that fit into the accelerator transverse normalized acceptance of $A_{T}=30 \mathrm{~mm} \mathrm{rad}$ and normalized longitudinal acceptance of $A_{L}=150 \mathrm{~mm}$. The 80-m-long cooling channel raises this quantity by about a factor of 1.7. The current best value is $0.176 \pm 0.006$ muons per incident proton. This is the same value obtained in FS2. Thus, we have achieved the identical performance at the entrance to the accelerator as FS2, but with a significantly simpler, shorter, and presumably less expensive channel design (see Sec. VII). In addition, unlike FS2,
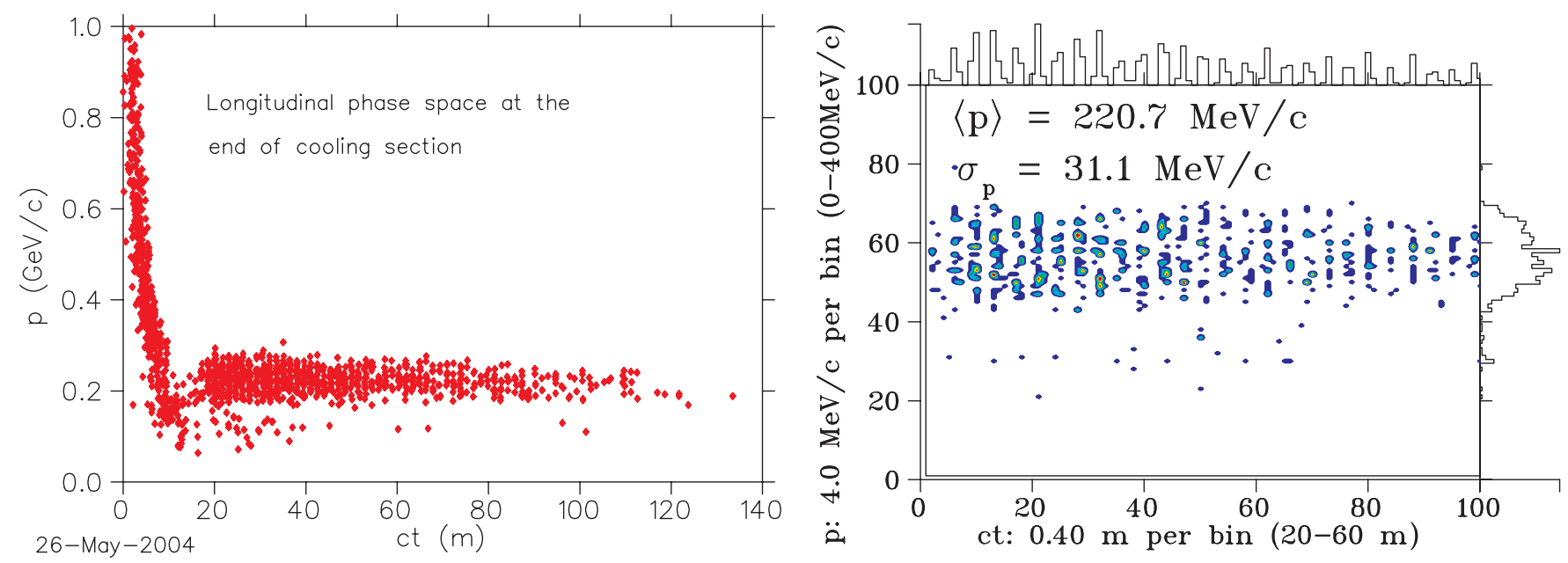

FIG. 13. (Color) Longitudinal phase space at the end of the channel. Right: the vertical scale expands from 0 to $400 \mathrm{MeV} / c$ with 100 bins; the horizontal scale is from 20 to $60 \mathrm{~m}$ with 100 bins.
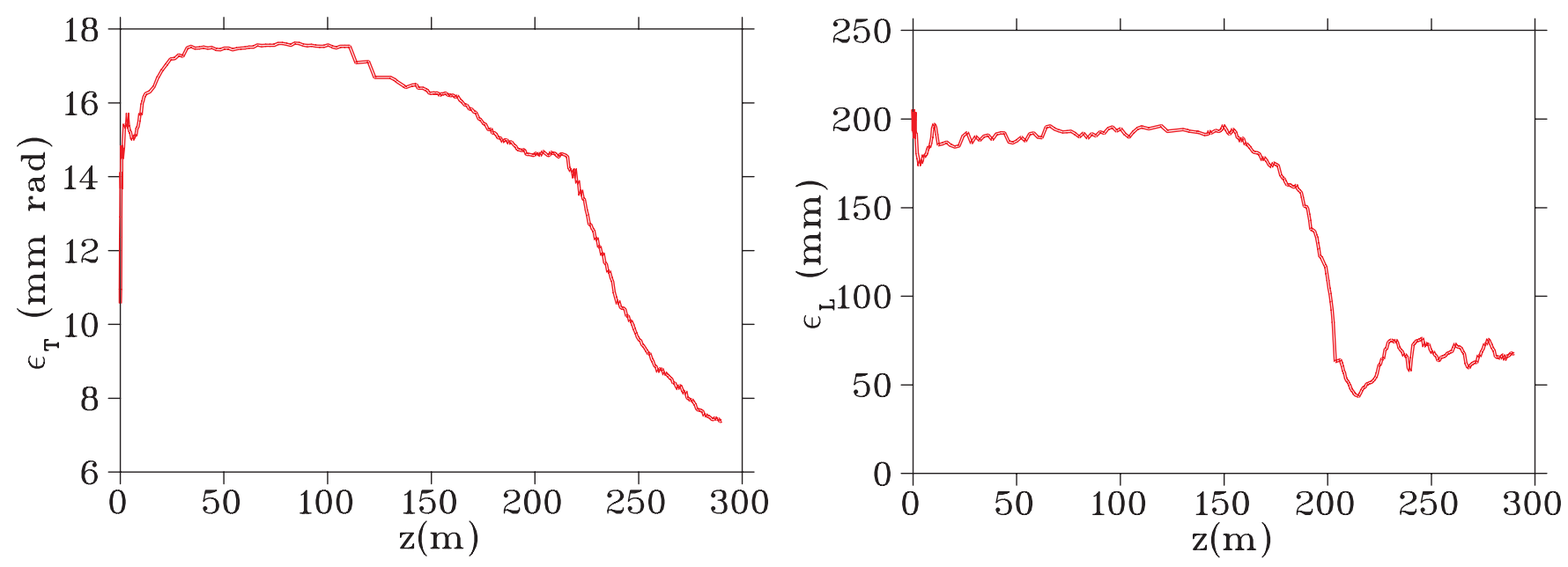

FIG. 14. (Color) Normalized transverse emittance (left) and longitudinal emittance (right) along the front end for a momentum cut $0.1 \leq p \leq 0.3 \mathrm{GeV} / c$. 


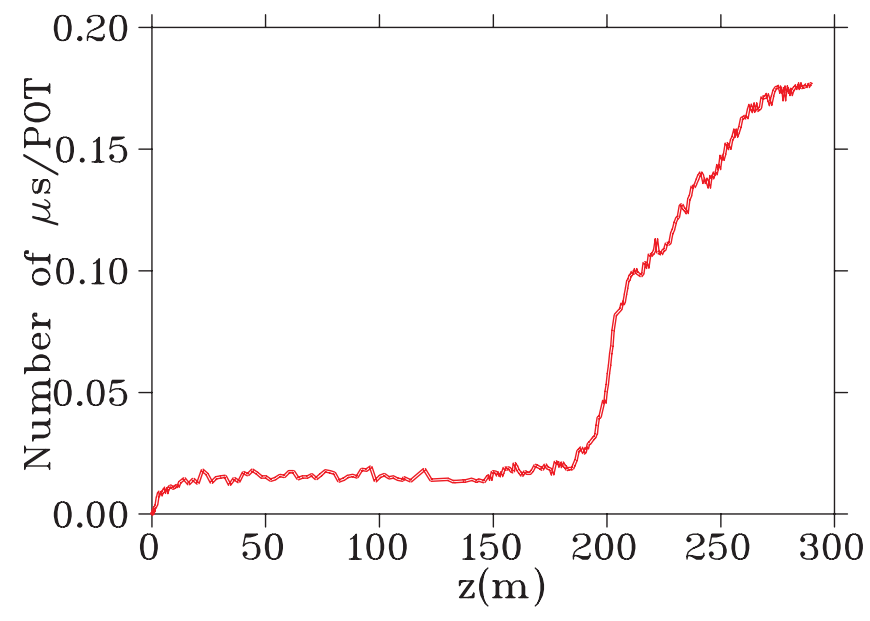

FIG. 15. (Color) The muons per incident proton on target into the accelerator normalized transverse acceptance of $A_{T}=$ $30 \mathrm{~mm} \mathrm{rad}$ and normalized longitudinal acceptance of $A_{L}=$ $150 \mathrm{~mm}$ for a momentum cut $0.1 \leq p \leq 0.3 \mathrm{GeV} / c$.

this channel transmits both signs of muons produced at the target. With appropriate modifications to the transport line going into the storage ring and the storage ring itself, this design could deliver both (time tagged) neutrinos and antineutrinos to the detector. The beam at the end of the cooling section consists of a train of bunches with a varying population of muons in each one; this is shown in Fig. 16 for one sign.

Figure 17 depicts the longitudinal phase space of the superposition of all bunches projected onto a single period $(T \approx 5 \mathrm{~ns})$. We assume that particles outside the accelerator acceptance are intercepted by collimators located in the matching section, although this collimation system has not been designed yet. Approximately $60 \%$ of the beam leaving the cooling channel is intercepted by the collimators.

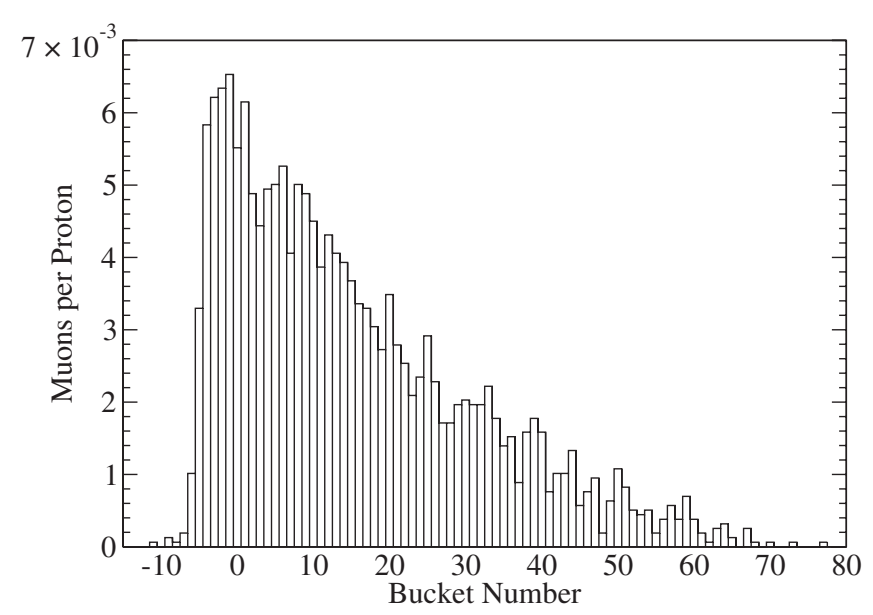

FIG. 16. Bunch structure of the beam delivered to the accelerator normalized transverse acceptance of $A_{T}=30 \mathrm{~mm}$ rad and normalized longitudinal acceptance of $A_{L}=150 \mathrm{~mm}$ for a momentum cut $0.1 \leq p \leq 0.3 \mathrm{GeV} / c$.

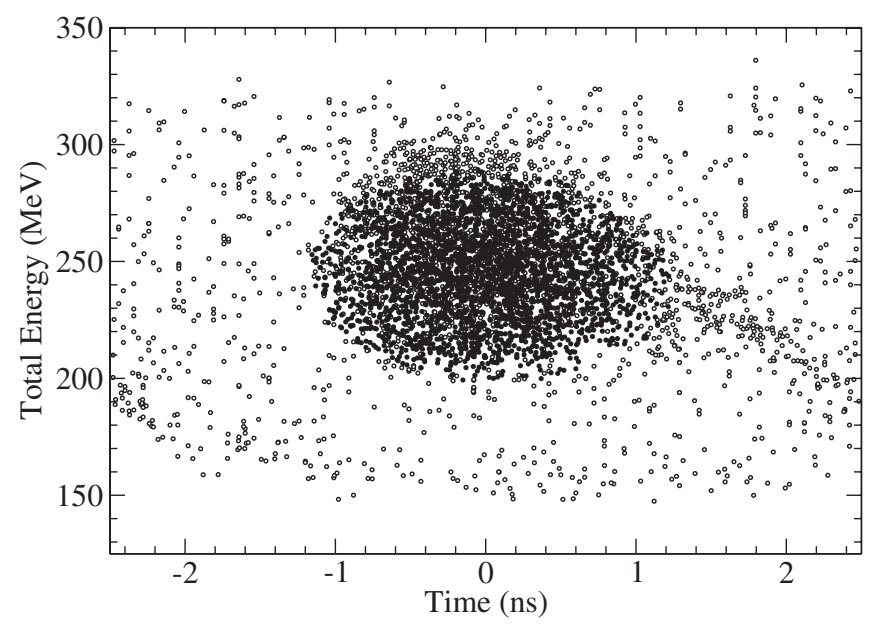

FIG. 17. Longitudinal phase space of overlayed bunches in the train at the end of the cooling section. The open circles are all the particles that reach the end of the channel and the filled circles are particles within the accelerator normalized transverse acceptance of $A_{T}=30 \mathrm{~mm}$ rad and normalized longitudinal acceptance of $A_{L}=150 \mathrm{~mm}$ for a momentum cut $0.1 \leq p \leq$ $0.3 \mathrm{GeV} / c$.

The heat load on the collimator is $7.3 \mathrm{~kW}$. Figure 18 shows a few interleaved $\mu^{+}$and $\mu^{-}$bunches exiting the cooling section. The opposite-sign bunches are mostly separated in time. There are a small number of wrong sign particles in the bunch train after cooling, but these will be cleanly separated by the dipoles in the subsequent accelerators and storage ring.

\section{Heating of absorber windows}

There are some unresolved issues with the absorber windows that will require further research and develop-

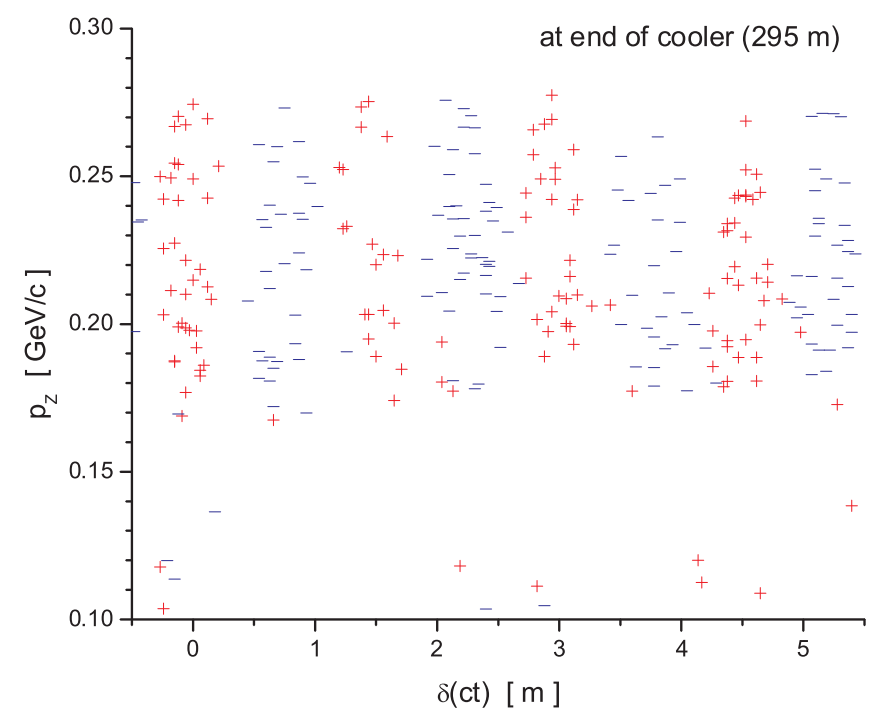

FIG. 18. (Color) A sample from the train of interleaved $\mu^{+}$(red) and $\mu^{-}$(blue) bunches exiting the cooling section. 
ment. To minimize multiple scattering we have assumed the windows are made from $\mathrm{LiH}$. In order to protect the $\mathrm{LiH}$ from the environment and to provide a high conductivity surface to close off the rf cavity, we have assumed the $\mathrm{LiH}$ is encased in a thin layer of Be. Assuming that the Be can be bonded to the $\mathrm{LiH}$, there is the question of what happens when the window is heated by energy loss of the muon beam and by the power deposited by the rf cavity. If the heating becomes high enough, melting and differential stresses leading to buckling are possible. In addition the window could suffer degradation from radiation damage.

Approximately $1.1 \times 10^{14}$ muons of each charge enter the start of the cooling channel each second. This produces a total power deposition of $\approx 58 \mathrm{~W}$ distributed along the beam path. Most of the energy deposition takes place in the $\mathrm{LiH}$. We assume that cooling is provided by a heat sink at the outer edge of the window. If we ignore any longitudinal heat conduction between the $\mathrm{LiH}$ and Be layers [20], the $\mathrm{LiH}$ reaches a maximum temperature of $310^{\circ} \mathrm{C}$ in steady state. This is safely below its melting temperature of $690^{\circ} \mathrm{C}$. The rf heating occurs in a skin depth on the side of the window facing the cavity. The skin depth for Be at $201 \mathrm{MHz}$ is approximately $9 \mu \mathrm{m}$. The rf power deposited on the window of a pillbox rf cavity is

$$
P=\frac{\pi^{2}}{2} \frac{d}{\lambda} E_{0}^{2} \frac{b^{2}}{Z_{0}}\left\{J_{1}^{2}(\alpha)-J_{0}(\alpha) J_{2}(\alpha)\right\}
$$

where, $d=$ skin depth, $\lambda=$ rf wavelength, $E_{0}=$ peak $\mathrm{rf}$ gradient, $b=$ windows radius, $a=$ radius of rf cavity (pillbox), $Z_{0}=$ impedance of free space, and $J_{0}, J_{1}, J_{2}$ are Bessel functions with argument $\alpha=2.405 \times \frac{b}{a}$. This gives a total $\mathrm{rf}$ power of $\approx 220 \mathrm{~W}$ in each window. Rough calculations predict that the temperature at the center of the $300 \mu \mathrm{m}$ thick Be layer should be less than $175^{\circ} \mathrm{C}$. This is also safely below its melting temperature of $1275^{\circ} \mathrm{C}$.

Although melting will not be a problem, buckling and delamination of the Be layer is a potential deleterious outcome. More accurate finite element thermal studies need to be done of the composite $\mathrm{LiH}-\mathrm{Be}$ system. In case this window design does not prove to be feasible, a number of alternative absorber designs have been investigated. The window could be cooled by flowing $\mathrm{He}$ gas between the Be window and the $\mathrm{LiH}$. The thermal conductivity to the heat sink on the outer edge of the window can be improved by breaking up the $\mathrm{LiH}$ into several pieces, separated by layers of high conductivity Be. Using a total thickness that gives the same total energy loss as the original window results in only $\approx 3 \%$ loss in the accepted muon flux. Other possibilities that gave reasonable muon fluxes are a thin Be layer on pure lithium or thicker Be windows and no $\mathrm{LiH}$, with a thickness chosen to make the total energy loss the same as that in the baseline $\mathrm{LiH}$ absorber case. Cooling would be a bit less effective because of the greater multiple scattering. An initial evaluation [18] of a Be-only scenario showed less capture into the acceleration channel acceptance $(\approx$
15\%). A scenario in which Be absorbers are initially installed and then upgraded later to more efficient $\mathrm{LiH}$ absorbers is, of course, also possible.

\section{ACCELERATION DESIGN}

The acceleration system takes the beam from the end of the cooling channel and accelerates it to the energy required for the decay ring. Figure 19 shows a compact potential layout for all the acceleration systems described here. It includes five subsystems: a matching section, a linac, a recirculating linear accelerator (RLA), and two FFAG circular accelerators.

To reduce costs, the RLA acceleration systems from FS2 [1] will be replaced, as much as possible, by FFAG accelerators. FFAGs are rings that accelerate a beam over a large energy range (generally at least a factor of 2) without varying the magnets' fields, allowing for very rapid acceleration. Since they are rings, the bunches make multiple passes through the rf cavities, reducing the rf voltage required to accelerate. The number of turns is not limited by the switchyard, as it is in an RLA. The original FFAG designs [21] ("scaling" FFAGs) used large, highly nonlinear magnets. For our design, we instead use so-called linear nonscaling FFAGs $[22,23]$. These FFAGs use very linear magnets to maximize the dynamic aperture (necessary for our large-emittance beams), and the magnets generally have smaller apertures than those in a corresponding scaling FFAG design, bringing down the machine cost.

Table III gives the design parameters of the acceleration system. Acceptance is defined such that if $A_{\perp}$ is the transverse acceptance and $\beta_{\perp}$ is the beta function, then the maximum particle displacement (of the particles we transmit) from the reference orbit is $\sqrt{\beta_{\perp} A_{\perp} m c / p}$, where $p$ is the particle's total momentum, $m$ is the particle's rest mass, and $c$ is the speed of light. The acceleration system is able to accelerate bunch trains of both signs simultaneously.

\section{A. Matching from cooling to acceleration linac}

The cooling section has a beta function of around $0.8 \mathrm{~m}$, whereas the beginning of the acceleration linac has a beta function of around $2.7 \mathrm{~m}$. A matching section is required to gradually change the beta functions from one section to the other so as to avoid emittance growth and/or particle loss.

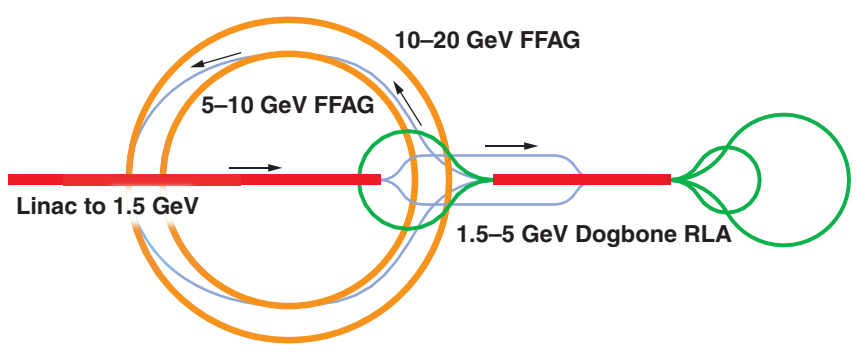

FIG. 19. (Color) Potential layout for the acceleration systems. 
TABLE III. Acceleration system design parameters.

\begin{tabular}{lc}
\hline \hline Injection momentum $(\mathrm{MeV} / c)$ & 273 \\
Initial kinetic energy $(\mathrm{MeV})$ & 187 \\
Final total energy $(\mathrm{GeV})$ & 20 \\
Normalized transverse acceptance $(\mathrm{mm})$ & 30 \\
Normalized transverse emittance, $\mathrm{rms}(\mathrm{mm} \mathrm{rad})$ & 3.84 \\
Normalized longitudinal acceptance, $\Delta E \Delta t / m_{\mu} c(\mathrm{~mm})$ & 150 \\
Total energy spread, $\Delta E(\mathrm{MeV})$ & \pm 45.8 \\
Bunch length (ns) & \pm 1.16 \\
Energy spread, rms $(\mathrm{MeV})$ & 19.8 \\
Bunch length, rms $(\mathrm{ns})$ & 0.501 \\
Bunching frequency $(\mathrm{MHz})$ & 201.25 \\
Maximum muons per bunch & $1.1 \times 10^{11}$ \\
Muons per bunch train $($ each charge) & $3.0 \times 10^{12}$ \\
Bunches in train & 89 \\
Average repetition rate $(\mathrm{Hz})$ & 15 \\
Minimum time between pulses (ms) & 20 \\
Average beam power at the end (each charge) $(\mathrm{kW})$ & 144 \\
\hline \hline
\end{tabular}

Furthermore, the reduced acceptance of the longer cells in the acceleration linac, as compared to the more compact cells of the cooling section, necessitates that the acceleration linac start at an energy above that of the cooling section (see Table III); the matching section will thus also begin to increase the beam energy after the cooling. That matching section will consist of six cells similar to those in the cooling channel, but with increasing lengths and numbers of cavities per cell, and three superconducting cells similar to the accelerating linac, but made shorter by the use of shorter, higher field focusing solenoids. Figure 20 shows a layout of the matching section. The current design for the matching section has about $15 \%$ loss; initial studies indicate that this may be due to performing the matching at low instead of high amplitudes. Initial attempts at performing the longitudinal match at high amplitudes have eliminated the losses longitudinally, but we have not yet done the matching for the transverse plane as well.

\section{B. Low energy acceleration}

Based on preliminary cost considerations, we have chosen not to use FFAGs below $5 \mathrm{GeV}$ total energy. Therefore, we must provide alternative acceleration up to that point. Similarly to what was adopted in FS2, we use a linac from the lowest energies to $1.5 \mathrm{GeV}$, followed by a recirculating linear accelerator (RLA).

The linac parameters are strongly constrained by the transverse acceptance. In FS2 there were three types of cryomodules, containing one, two, and four two-cell cav-

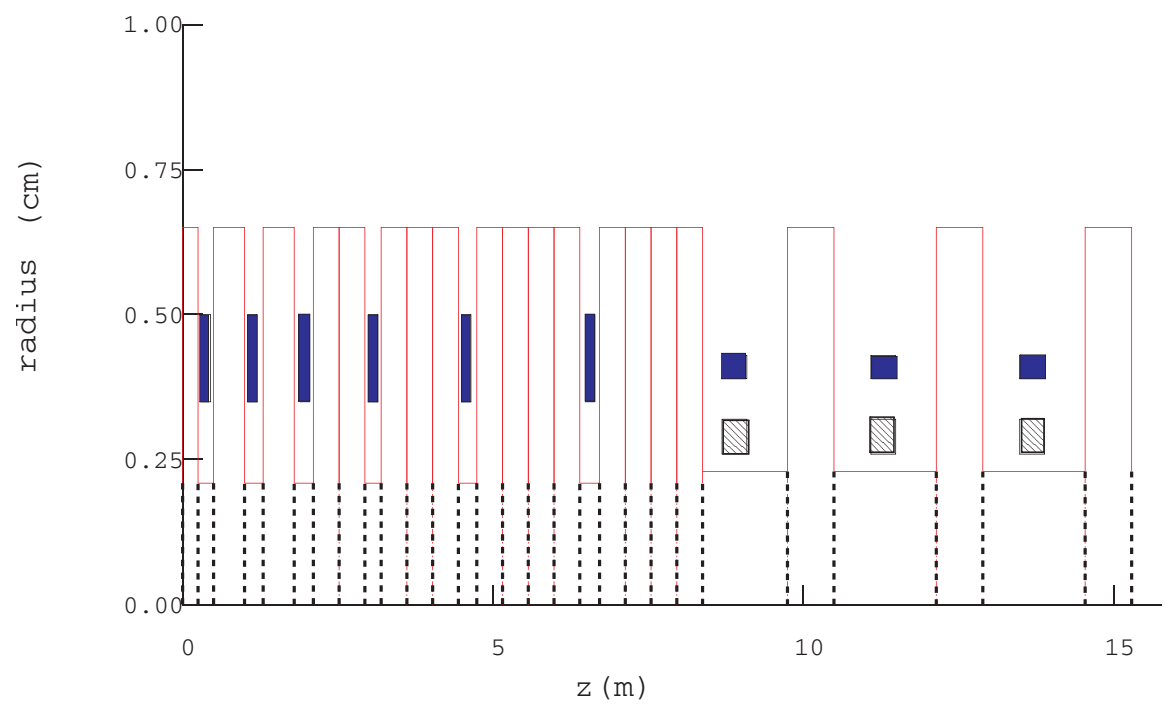

FIG. 20. (Color) Matching section from cooling to linac. Blue rectangles are solenoids, red lines are rf cavity walls and dashed black lines are the windows of the cavities. The last three cavities are superconducting, the remaining ones are room temperature. 
ities, respectively. Because of our larger acceptance requirements, the cryomodule dimensions from FS2 would require the beam to have a momentum of at least 420,672 , and $1783 \mathrm{MeV} / c$, respectively. Note that the momentum for the first stage of the linac is, already, much higher than the average momentum in the cooling channel, which is about $220 \mathrm{MeV} / c$. Thus, we need to make adjustments to the FS2 design to be able to accelerate this larger beam.

In particular, to increase the acceptance, we must reduce the lengths of the cryomodules. We first employ a very short cryomodule using a single one-cell cavity as opposed to the two-cell cavities used in all of the FS2 cryomodules. Not only does this shorten the cavity itself, it also eliminates one of the input couplers. We also eliminate some of the drift space in the cryomodule. This is possible since we now consider it acceptable to run the cavities with up to $0.1 \mathrm{~T}$ on them [24], provided the cavities are cooled down before the magnets are powered. The field profile of the solenoids shown in FS2 indicates that the iron shield on the solenoids is sufficient to bring the field down to that level, even immediately adjacent to the solenoid shield. Together, these changes reduce the total length for the first module type to only $3 \mathrm{~m}$. Table IV shows the dimensions of the cryostats we will use and Fig. 21 depicts all three of them.

Table V summarizes parameters for the linac. The phases of the cavities in the linac will be varied approximately linearly with length from about $-73^{\circ}$ at the beginning of the linac to $0^{\circ}$ at the end, as shown in Fig. 22.

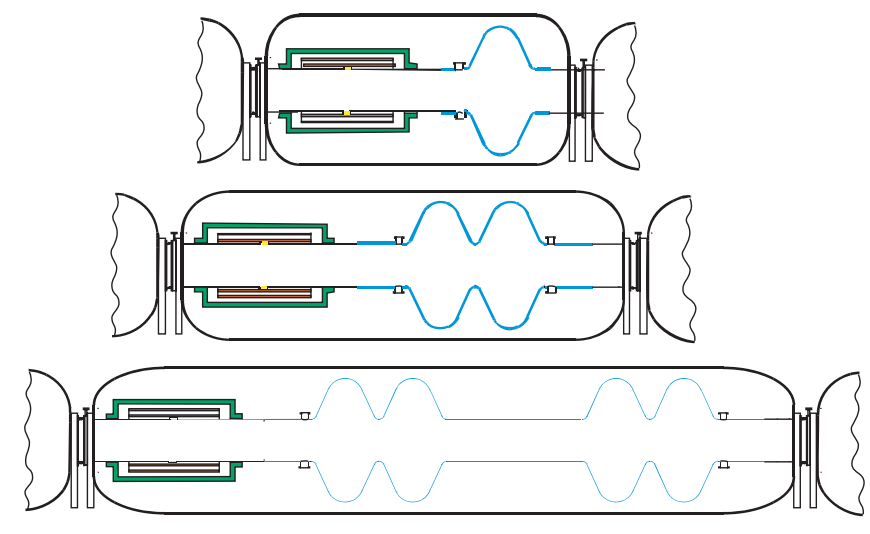

FIG. 21. (Color) Layouts of superconducting linac preaccelerator cryomodules. Blue lines are the SC walls of the cavities, solenoid coils are indicated in red, and the iron shielding is in green. The dimensions of the cryomodules are shown in Table IV, and Table V summarizes parameters for the linac.

\section{RLAs}

Compared with FS2, we are injecting into the RLA at a lower energy and are accelerating over a much smaller energy range. These features make it more difficult to have a large number of turns in the RLA. To mitigate this, we choose a dog-bone layout for the RLA [25]. For a given amount of installed rf, the dog-bone layout has twice the energy separation of the racetrack layout at the spreaders

TABLE IV. Linac cryomodule structure. Numbers are lengths in $\mathrm{m}$.

\begin{tabular}{cccccc}
\hline \hline \multicolumn{2}{c}{ Cryostat I } & \multicolumn{2}{c}{ Cryostat II } & \multicolumn{2}{c}{ Cryostat III } \\
\hline Drift & 0.45 & Drift & 0.70 & Drift & 0.70 \\
Solenoid & 1.00 & Solenoid & 1.00 & Solenoid & 1.00 \\
Drift & 0.50 & Drift & 1.00 & Drift & 1.00 \\
Cavity & 0.75 & Cavity & 1.50 & Cavity & 1.50 \\
Drift & 0.30 & Drift & 0.80 & Drift & 1.50 \\
Total & 3.00 & Total & 5.00 & Cavity & 1.50 \\
& & & & Drift & 0.80 \\
& & & & Total & 8.00 \\
\hline \hline
\end{tabular}

TABLE V. Linac cryomodule parameters.

\begin{tabular}{lccc}
\hline \hline & Cryo I & Cryo II & Cryo III \\
\hline Length (m) & 3.00 & 5.00 & 8.00 \\
Number of modules & 12 & 18 & 22 \\
Cells per cavity & 1 & 2 & 2 \\
Cavities per module & 1 & 1 & 2 \\
Maximum energy gain per cavity (MeV) & 11.2 & 22.4 & 22.4 \\
Cavity rf frequency (MHz) & 201.25 & 201.25 & 201.25 \\
Solenoid length (m) & 1 & 1 & 1 \\
Max Solenoid field (T) & 1.5 & 1.8 & 4.0 \\
\hline \hline
\end{tabular}




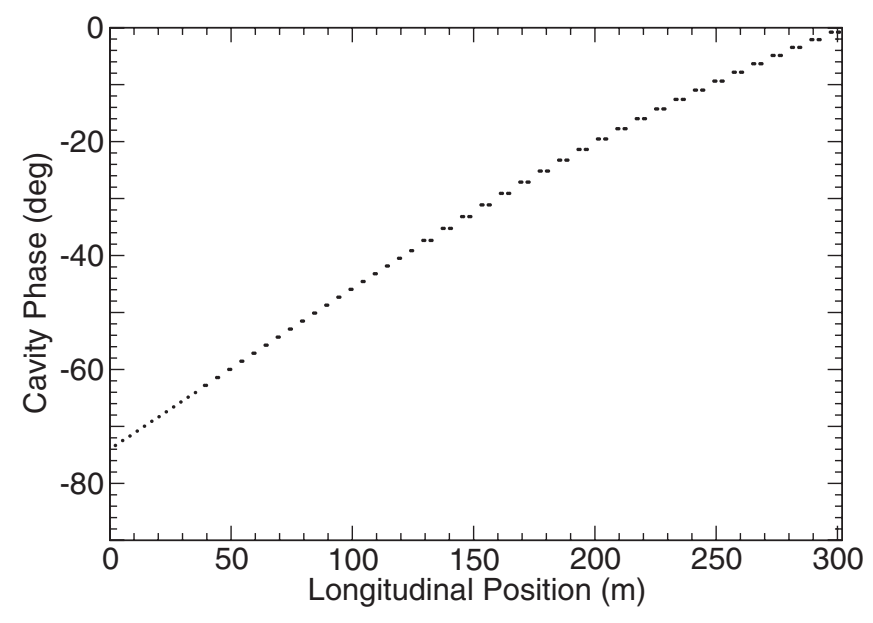

FIG. 22. Cavity phase vs position along the linac.

and recombiners (see Fig. 24), making the switchyard much easier and allowing more passes through the linac.

One disadvantage of the dog-bone layout is that, because of the longer linac and the very low injection energy, there is a significant phase shift of the reference particle with respect to the cavity phases along the length of the linac in the first pass, relative to later passes. To reduce this effect, we inject into the center of the linac as shown in Fig. 23. This injection is accomplished with a chicane similar to that used for injection in FS2, but here, to inject both signs, there are two chicanes, one on either side of the linac (see Fig. 25). The start of the chicanes is the point at which the particles with differing charges are first separated. To avoid this point overlapping the earlier part of the linac, the chicanes are tilted slightly upwards.

In the dog-bone RLA we have just over $1 \mathrm{GeV}$ of linac, and we make three and a half passes through that linac to accelerate from a total energy of $1.5 \mathrm{GeV}$ to $5 \mathrm{GeV}$. The RLA linac consists of $11 \mathrm{~m}$ long cells with two 2-cell cavities per cell, and quadrupole triplet focusing, as shown in Fig. 26. The cavities are the $30 \mathrm{~cm}$ aperture cavities assumed in FS2, as opposed to the $46 \mathrm{~cm}$ aperture cavities that were used in the linac that accelerated up to $1.5 \mathrm{GeV}$; this should permit a somewhat higher gradient $(17 \mathrm{MV} / \mathrm{m}$ rather than $15 \mathrm{MV} / \mathrm{m})$.

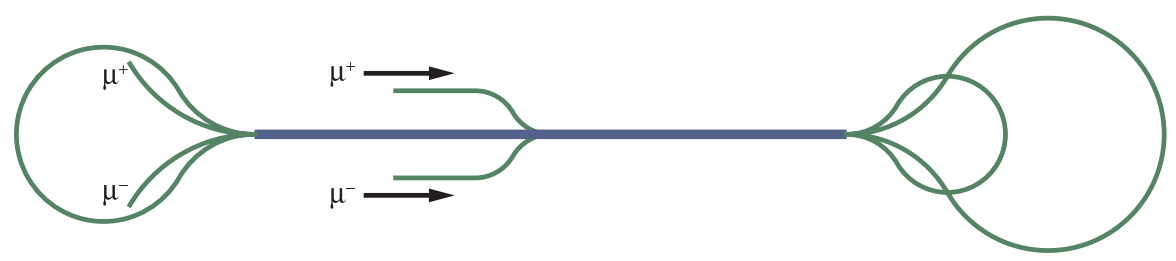

FIG. 23. (Color) Dog-bone (top) and racetrack (bottom) layout for the RLA.
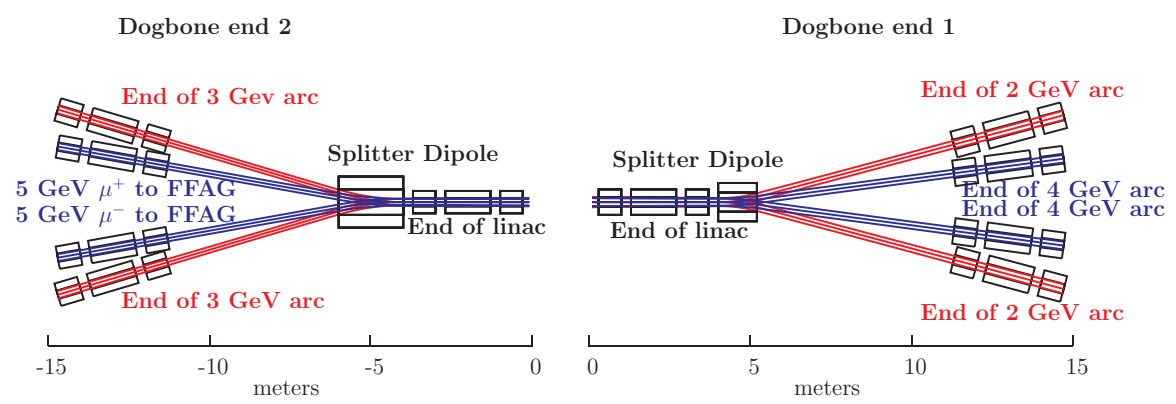

FIG. 24. (Color) Dog-bone RLA Switch Yards.

End Pre-Linac

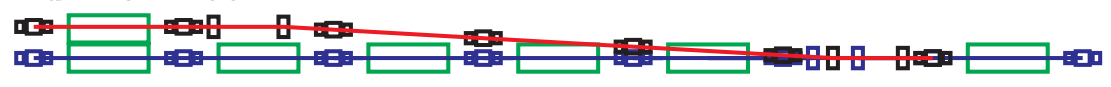

RLA Linac

Elevation

RLA Linac

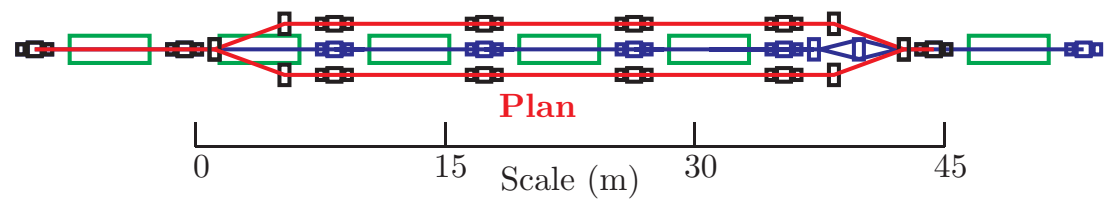

FIG. 25. (Color) Injection chicanes. Magnets in black, $r f$ in green, RLA linac in blue, and injection lines in red. 


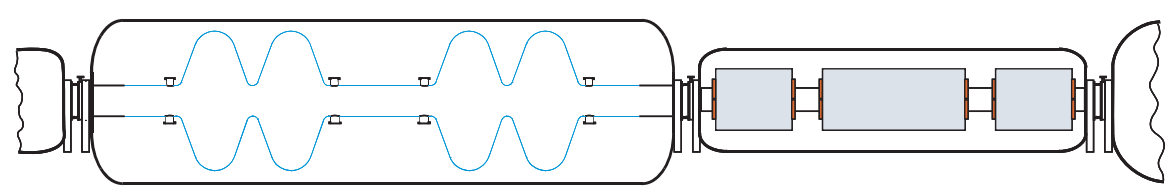

FIG. 26. (Color) Dog-bone linac cell.

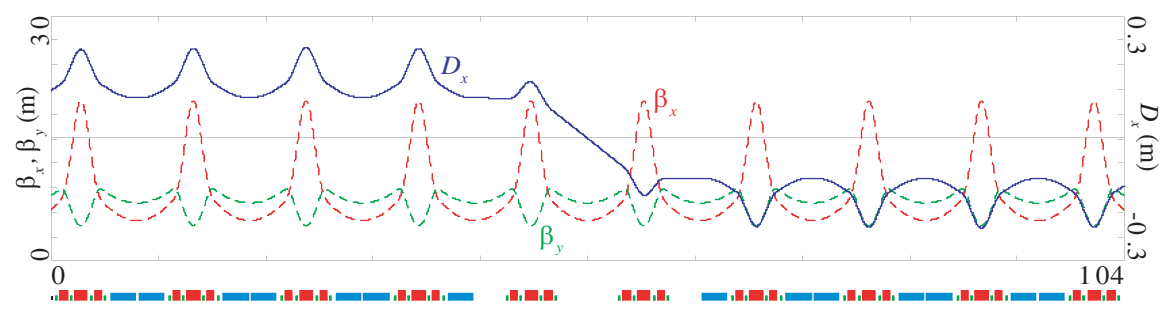

FIG. 27. (Color) A section of the dog-bone arc where the bend changes direction, showing the dispersion (solid) and beta functions (dashed).

The arcs will also use quadrupole triplet focusing, with a $90^{\circ}$ phase advance per cell in both planes, in order to cancel some chromatic effects. Both the quadrupoles and the dipoles in the arc and linac lattices will have $1 \mathrm{~T}$ maximum field at the coils, and can be warm magnets.

Since the dog-bone arc changes its direction of bend twice in each arc, dispersion matching must be handled carefully. This is done straightforwardly by having a $90^{\circ}$ phase advance per cell, and removing the dipoles from two consecutive cells. This will cause the dispersion to switch to the other sign as desired, as shown in Fig. 27. Matching of off-momentum particles is controlled using sextupoles.

\section{FFAGs}

Once we reach $5 \mathrm{GeV}$, it appears to be more costeffective to use FFAGs rather than RLAs. This conclusion is based on applying a procedure for producing minimumcost FFAG designs [26,27] and comparing the resulting costs to those from FS2. FFAG designs for accelerating from 5 to $10 \mathrm{GeV}$ and from 10 to $20 \mathrm{GeV}$ are given in Table VI. The lattices consist exclusively of combinedfunction triplet cells with a drift length sufficient to hold a single-cell 201.25 MHz superconducting rf cavity (similar to the double-cell cavities from FS2). The $2 \mathrm{~m}$ length of the drift is needed to keep the fields on the cavity under $0.1 \mathrm{~T}$ [24].

With the $1 \mathrm{MW}$ beam intensity given in Table III, and both signs of muons, about $16 \%$ of the stored energy will be extracted from the cavities in the 5-10 GeV FFAG, and about $27 \%$ will be extracted in the $10-20 \mathrm{GeV}$. While this may seem substantial, it is easily handled. To keep the average voltage sufficient to accelerate over the desired range, $7.5 \mathrm{MV}$, one need only increase the initial voltage to 7.8 MV for the 5-10 GeV FFAG and to $8.1 \mathrm{MV}$ for the 10$20 \mathrm{GeV}$ FFAG. The most important effect is a differential acceleration between the head and tail of the bunch train,

TABLE VI. Parameters for FFAG lattices. See Fig. 28 to understand the signs of the parameters.

Maximum energy gain per cavity $(\mathrm{MeV})$

Stored energy per cavity (J)

Cells without cavities

RF drift length (m)

Drift length between quadrupoles (m)

Initial total energy $(\mathrm{GeV})$

Final total energy $(\mathrm{GeV})$

Number of cells

Magnet type

Magnet length (m)

Reference orbit radius of curvature (m)

Magnet center offset from reference orbit (mm)

Magnet aperture radius $(\mathrm{cm})$

Field on reference orbit $(\mathrm{T})$

Field gradient $(\mathrm{T} / \mathrm{m})$
7.5

368

8

2

0.5

5

10

90

Defocusing

1.612338

15.2740

$-1.573$

14.0916

1.63774

$-9.1883$

.5
368
8
2
.5

\begin{tabular}{|c|c|c|}
\hline & & \\
\hline Focusing & Defocusing & Focusing \\
\hline 1.065600 & 1.762347 & 1.275747 \\
\hline-59.6174 & 18.4002 & -70.9958 \\
\hline 7.667 & 1.148 & 8.745 \\
\hline 15.2628 & 10.3756 & 12.6256 \\
\hline-0.41959 & 2.71917 & -0.70474 \\
\hline 8.1768 & -15.4948 & 12.5874 \\
\hline
\end{tabular}




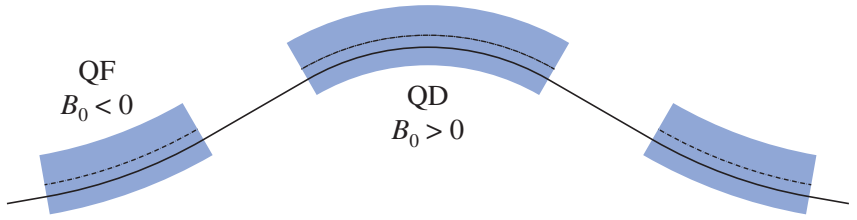

FIG. 28. (Color) Geometry of the triplet lattice. The "magnet center offset from reference orbit" listed in Table VI is positive for both magnets in this diagram.

which is only about $1 \%$ for both cases. This should be at least partially correctable by a phase offset between the cavity and the bunch train and, in any case, is substantially smaller than the energy spread in a single bunch.

One of the biggest challenges for the FFAGs is injection and extraction. Table VII gives the parameters required for injection and extraction kickers. The stored energy in the kicker is high, but is similar to that found in induction linac cells. The rise times and voltages are also similar to those in induction linacs. These parameters assume that injection occurs from the inside of the FFAG. This is preferred since the beam will be near the inside of the FFAG at the lowest energies. Figs. 29 and 30 show the $5 \mathrm{GeV}$ injection and $10 \mathrm{GeV}$ extraction layout. The magnets near the kickers and septum must be modified to accommodate the injection and extraction systems, but their effects will be kept as close as possible to those of the other cells in the FFAG lattice to minimize the driving of resonances.

\section{E. FFAG tracking results}

Initial experience with FFAG lattices having a linear midplane field profile has shown them to have a good dynamic aperture at fixed energies. We are careful to avoid single-cell linear resonances to prevent beam loss. However, since the tune is not constant, the single-cell tune will pass through many nonlinear resonances. Nonlinearities in the magnetic field due to end effects are capable of driving those nonlinear resonances, and we

TABLE VII. Parameters for FFAG injection and extracting kickers.

\begin{tabular}{lcccc}
\hline \hline Energy $(\mathrm{GeV})$ & 5 & 10 & 10 & 20 \\
Type & Inject & Extract & Inject & Extract \\
Length (m) & 1.5 & 1.5 & 1.5 & 1.5 \\
Kick field (T) & 0.37 & 0.51 & 0.78 & 0.58 \\
Maximum field at the coils (T) & 3.6 & 2.6 & 4.2 & 5.6 \\
Vertical aperture (cm) & 10 & 10 & 7.6 & 7.6 \\
Horizontal aperture (cm) & 25 & 25 & 19.5 & 19.5 \\
Current (kA) & 44 & 60 & 71 & 53 \\
Supply voltage (kV) & \pm 58 & \pm 60 & \pm 52 & \pm 48 \\
Rise/fall time (ns) & 640 & 950 & 875 & 1270 \\
Pulse length (ns) & 300 & 300 & 300 & 300 \\
Stored energy (J) & 850 & 1620 & 2280 & 1260 \\
\hline \hline
\end{tabular}

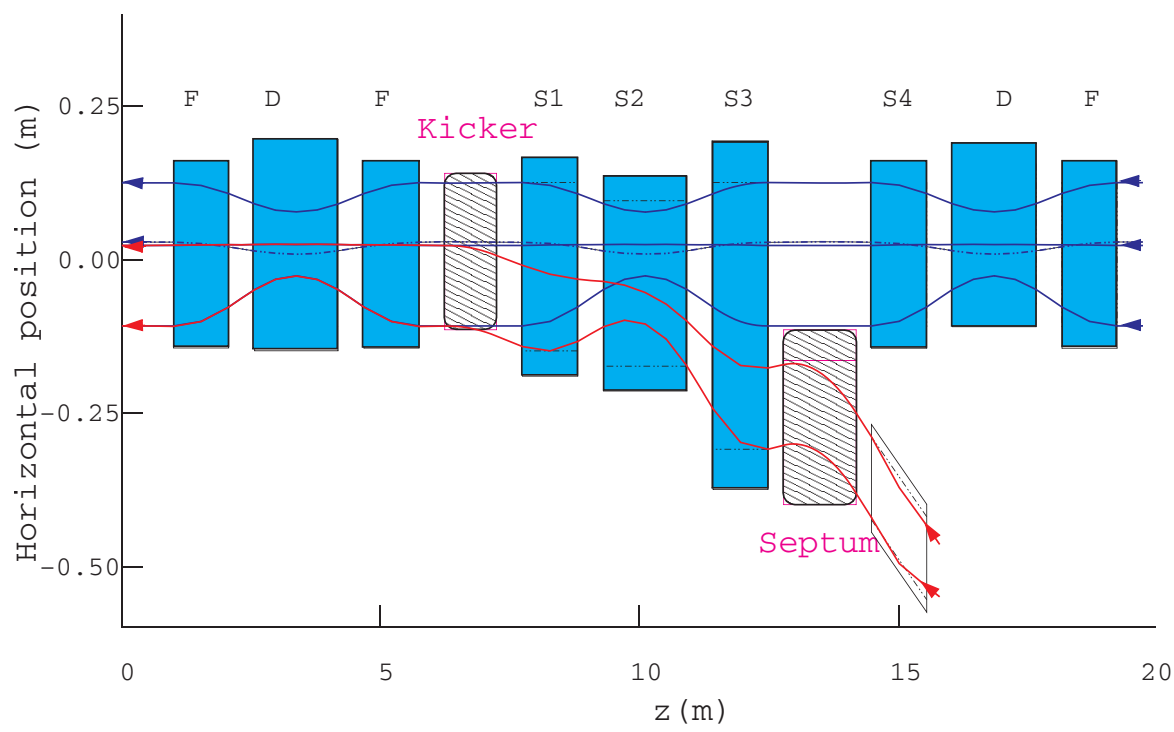

FIG. 29. (Color) Injection into the FFAG. S1 to S4 are special injection region dipoles. 


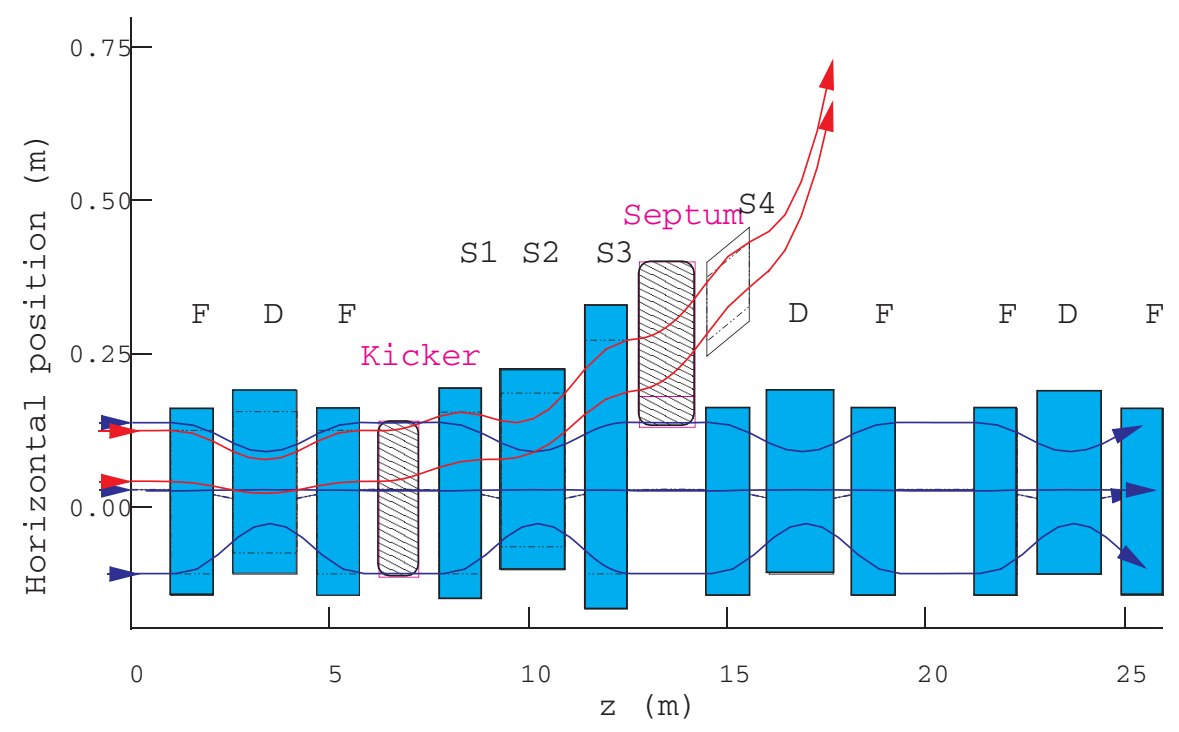

FIG. 30. (Color) Extraction out of the FFAG. S1 to S4 are special extraction region dipoles.

must be sure that there is no beam loss and minimal emittance growth because of this. Furthermore, there is the potential to weakly drive multicell linear resonances because the changing energy makes subsequent cells appear slightly different from each other. These effects can be studied through tracking.

ICOOL [15] is used for tracking for several reasons. It allows a fairly arbitrary end-field description, it forces that description to be consistent with Maxwell's equations, and it will track accurately even when the lattice acceptances, beam sizes, and energy spread are all large.

We begin by constructing a simple model of both a quadrupole and dipole $\cos \theta$-type magnet, without iron, using TOSCA [28]. At the end of the magnet, the field does not immediately drop to zero, but falls gradually, as shown in Fig. 31. The end-field falloff in a dipole or a quadrupole generates nonlinear fields, which ICOOL calculates. In addition, there are higher-order multipoles generated by breaking the magnet symmetry at the ends where

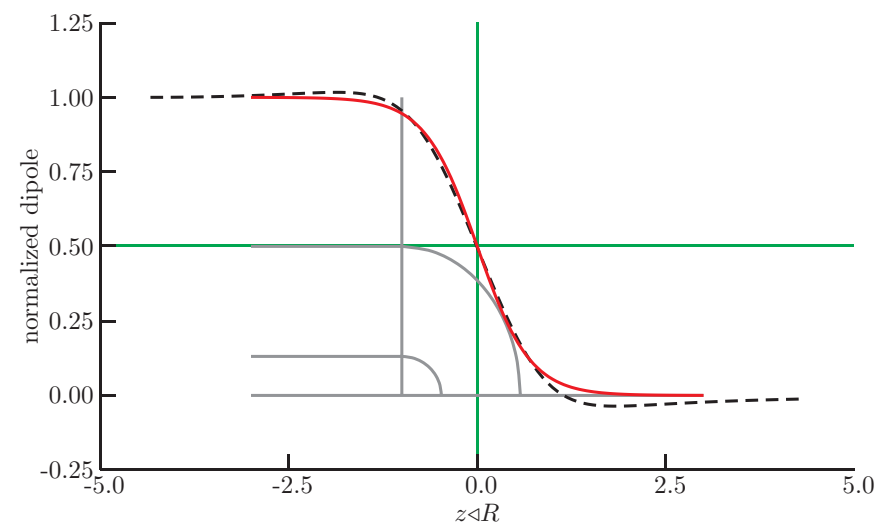

the coils form closed loops. We use TOSCA to compute the sextupole components that arise from this effect, as shown in Fig. 32, and include them in our computation.

The TOSCA computation is done without iron, which leads to the overshoot in the field values in Figs. 31 and 32. Iron in the magnet will likely eliminate that overshoot. Thus, we approximate the fields from TOSCA using functions without the overshoot. Fitting roughly to the TOSCA results, the fields are approximated by

$$
\begin{gathered}
B_{0}(z)=\frac{1}{2} B_{00}\left(1+\tanh \frac{z}{0.7 R}\right), \\
B_{1}(z)=\frac{1}{2} B_{10}\left(1+\tanh \frac{z}{0.35 R}\right) \\
B_{2}(z)=-0.2 B_{00} \exp \left[-\frac{1}{2}\left(\frac{z-0.36 R}{0.57 R}\right)^{2}\right],
\end{gathered}
$$

where $R$ is the magnet aperture radius, $B_{0}(z)$ is the dipole

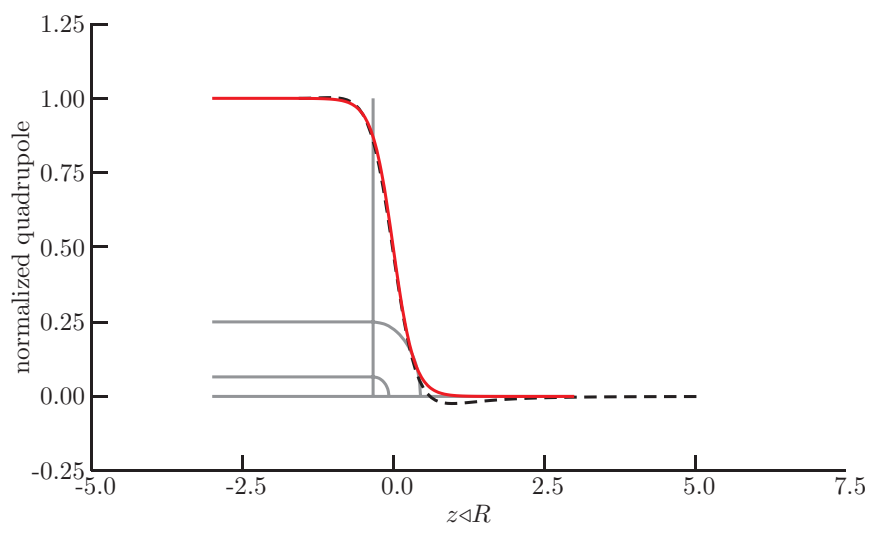

FIG. 31. (Color) Relative dipole field (left) and quadrupole field (right) near the magnet end. The dashed line is the field from TOSCA, while the solid line is our model. 


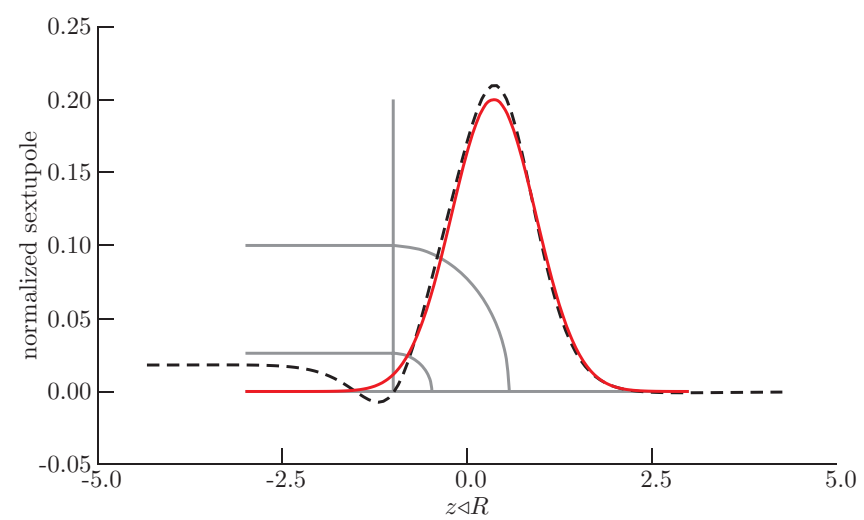

FIG. 32. (Color) Peak magnitude of the sextupole end field at radius $R$ (the magnet aperture), divided by the dipole field. The dashed line is the field from TOSCA, while the solid line is our model.

field, $B_{00}$ is the dipole field in the center of the magnet, $B_{1}$ is the quadrupole field, $B_{10}$ is the quadrupole field in the center of the magnet, and $B_{2}$ is the maximum magnitude of the sextupole field at the radius $R$. These fitted functions are shown in red in their corresponding plots in Figs. 31 and 32 .

Injecting particles at the outer edge of the acceptance and tracking through several cells indicated a large thirdorder resonance at around $5.1 \mathrm{GeV} / c$, as shown in Fig. 33 . This resonance is presumably being driven by the sextupole fields at the magnet ends. With some experimentation, it was found that if the integrated body sextupole was set to $68 \%$ of the integrated end sextupoles, (see Fig. 34), the resonance was eliminated (also shown in Fig. 33). When acceleration is included, one sees particle loss when accelerating through the resonance if there is no body sextupole correcting the end sextupoles, while there appears to be almost no loss with the body correction included (see

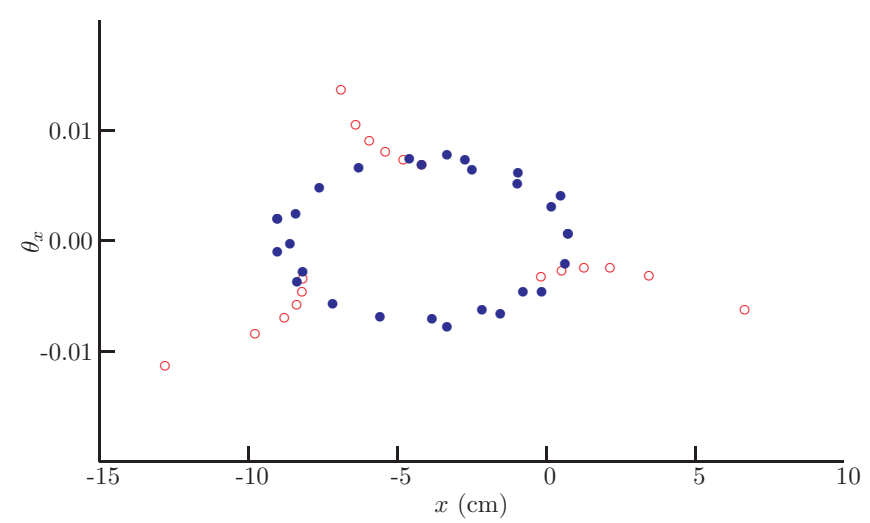

FIG. 33. (Color) Horizontal phase space from tracking at $5.1 \mathrm{GeV} / c$ at the outer edge of the acceptance. Open circles are without the body sextupole fields and show a third-order resonance; filled circles are with the body sextupole fields.

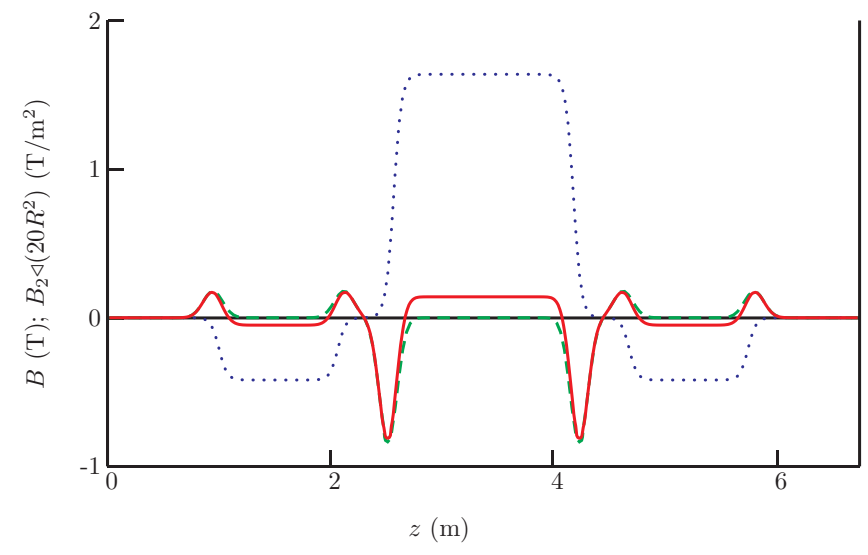

FIG. 34. (Color) Sextupole field components in the lattice used for tracking. The dotted line is the dipole field, the dashed line is $B_{2} /\left(20 R^{2}\right)$ with zero body sextupole field, and the solid line is with sufficient body sextupole field to eliminate the third-order resonance.

Fig. 35). If the body correction is only partially included, there is significant emittance growth. With these sextupole corrections, we can uniformly accelerate over the entire 5$10 \mathrm{GeV}$ energy range without losing a high-amplitude particle or having its amplitude grow by a large amount.

The $10-20$ GeV FFAG should have qualitatively similar and quantitatively better performance when tracked since (i) the tune vs energy profile of the machines is similar, (ii) the $10-20 \mathrm{GeV}$ machine has more cells than the 5-10 GeV machine, reducing the nonlinearities arising from curvature effects, and (iii) the ratio of aperture to length in the magnets is smaller for the $10-20 \mathrm{GeV}$ machine, reducing the relative contribution of the magnet end fields.

When tracking with rf is considered, the longitudinal dynamics is complex [29]. If one begins with an upright ellipse, there is considerable emittance growth if only the 201.25 MHz rf is used (see left plot in Fig. 36). Adding a

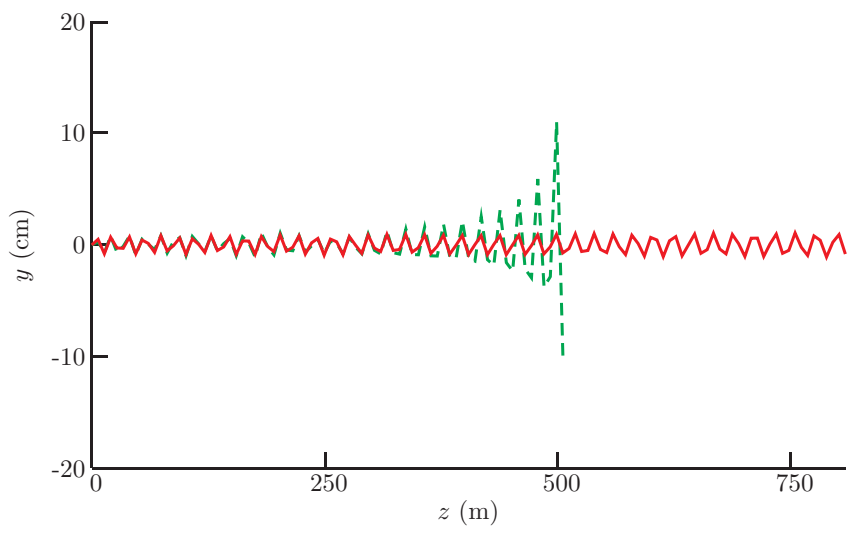

FIG. 35. (Color) Tracking of a particle at the edge of the acceptance with uniform acceleration. The dashed line is without any body sextupole, and the solid line is with the corrected body sextupole. 

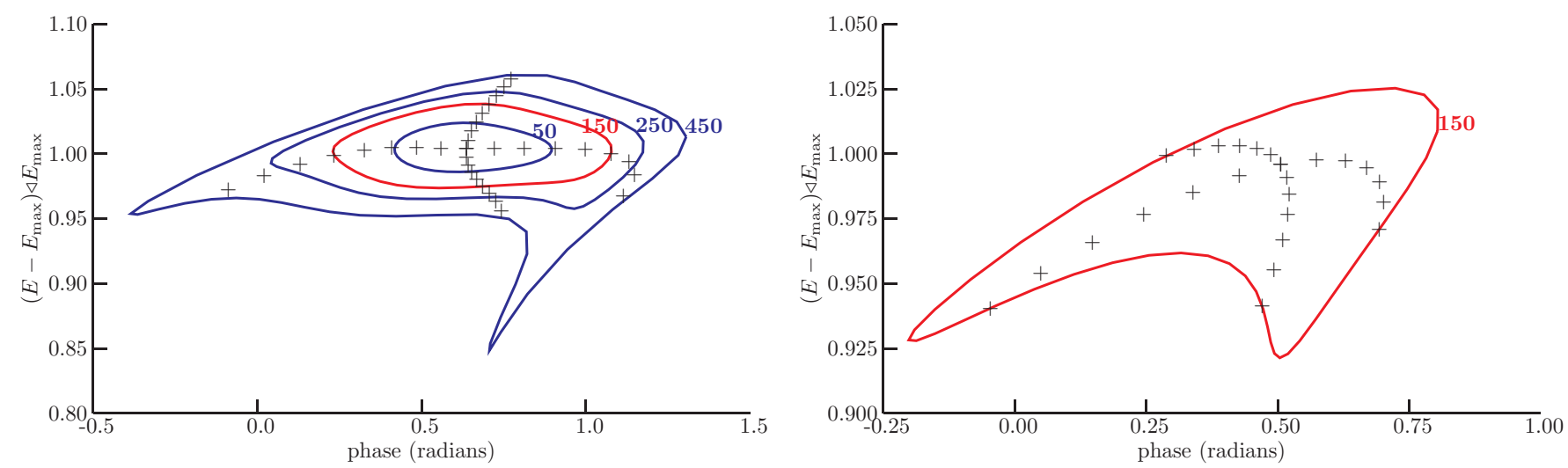

FIG. 36. (Color) Longitudinal tracking starting from an upright ellipse for the 5-10 GeV FFAG. On the left with only $201.25 \mathrm{MHz}$ rf. On the right with third-harmonic rf having voltage equal to $2 / 9$ of the fundamental rf voltage. Curves are labeled with their corresponding acceptance. Crosses for both cases started out as horizontal and vertical lines in phase space.

third-harmonic rf considerably reduces the emittance growth, as shown in right plot in Fig. 36. The amount of third-harmonic rf required is substantial and that, combined with space considerations, makes this alternative unattractive. An alternative that includes tilting the initial ellipse in phase space, which also reduces the emittance growth, is being studied.

\section{F. Design of combined-function superconducting mag- net for FFAGs}

An initial design of a superconducting combinedfunction (dipole-quadrupole) magnet has been developed [30]. The work has been done for the defocusing magnet from the above design. The parameters of this QD combined-function magnet are shown in Table VIII.

The magnet design is based on a cosine-theta configuration with two double layers for each function. A cross section for one quadrant is shown in Fig. 37. The quadrupole coil is located within the dipole coil and both coils are

TABLE VIII. Parameters of the QD magnet: $L_{0}$ is the length of the long drift between the QF magnets; $L_{q}$ is the length of the short drift between QF and QD magnets; $X_{0}$ is the displacement of the center of the magnet from the reference orbit (see Fig. 28); $B_{0}$ is the vertical magnetic field at the reference orbit, and $B_{1}$ is the derivative of the vertical magnetic field at the reference orbit.

\begin{tabular}{lc}
\hline \hline Initial energy, $E_{\min }(\mathrm{GeV})$ & 10 \\
Final energy, $E_{\max }(\mathrm{GeV})$ & 20 \\
Long drift, $L_{0}(\mathrm{~m})$ & 2 \\
Short drift, $L_{q}(\mathrm{~m})$ & 0.5 \\
Type of magnet & QD \\
Length of reference orbit, $L(\mathrm{~m})$ & 1.762 \\
Radius of curvature, $r(\mathrm{~m})$ & 18.4 \\
Displacement, $X_{0}(\mathrm{~mm})$ & 1.148 \\
Radius of the magnet bore, $R(\mathrm{~cm})$ & 10.3756 \\
Vertical magnetic field, $B_{0}(\mathrm{~T})$ & 2.7192 \\
Gradient, $B_{1}(\mathrm{~T} / \mathrm{m})$ & -15.495 \\
\hline \hline
\end{tabular}

assembled using key-and-bladder technology. All coils are made with the same $\mathrm{Nb}-\mathrm{Ti}$ cable capable of generating the operating dipole field and gradient with about the same current of $1800 \mathrm{~A}$. A single power supply is thus possible with a bit of fine tuning. The maximum central dipole field and gradient at short sample are $4.1 \mathrm{~T}$ and $26 \mathrm{~T} / \mathrm{m}$, as compared with the requirements of $2.7 \mathrm{~T}$ and $15.4 \mathrm{~T} / \mathrm{m}$, respectively. At this early design stage, excess margin is left for safety and perhaps a field rise in the magnet end region. The maximum azimuthal forces required for magnet prestress are of the order of $1 \mathrm{MN} / \mathrm{m}$ (assuming maximum safety). The conductor strand size and cable parameters common to both dipole and quadrupole are listed in Table IX.

The initial cross sections of both dipole and quadrupole were designed to give less than 0.01 units of systematic

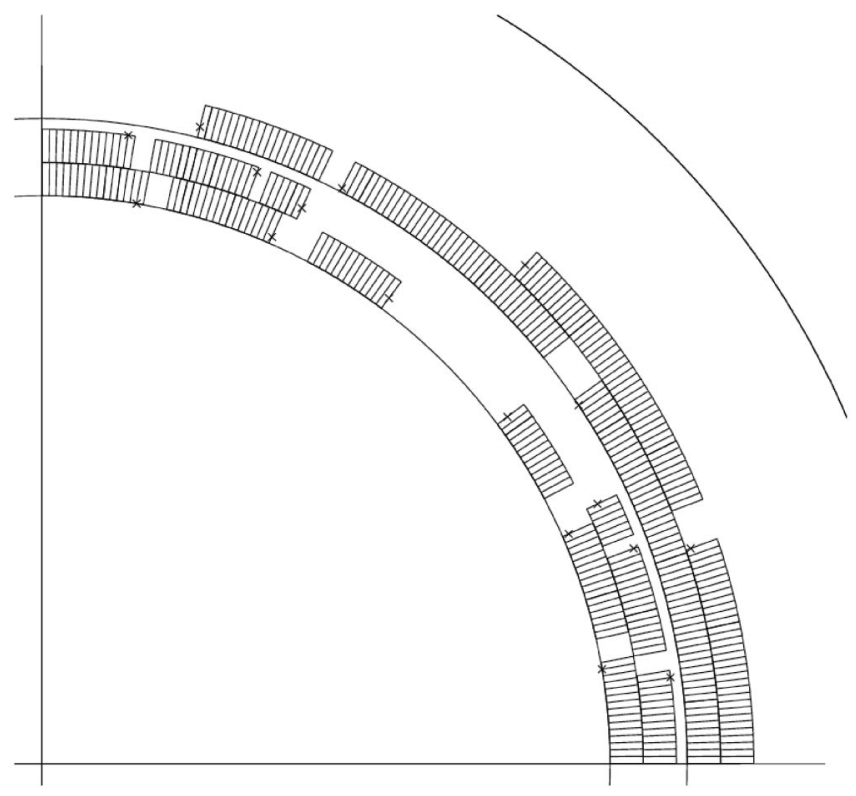

FIG. 37. First quadrant of the combined-function magnet cross section. 
TABLE IX. $\mathrm{Nb}-\mathrm{Ti}$ conductor for dipole and quadrupole coils.

\begin{tabular}{lc}
\hline \hline Strand diameter $(\mathrm{mm})$ & 0.6477 \\
Cable width, bare $(\mathrm{mm})$ & 6.4 \\
Cable thickness, insulated $(\mathrm{mm})$ & 1.35 \\
Keystone angle (deg) & 0.6814 \\
Conductor type & $\mathrm{Nb}-\mathrm{Ti}$ \\
Cu:SC ratio & $1.8: 1$ \\
Current density (at $5 \mathrm{~T}, 4.2 \mathrm{~K})\left(A / \mathrm{mm}^{2}\right)$ & 2850 \\
Number of strands & 20 \\
\hline \hline
\end{tabular}

multipole errors at a radius of $70 \mathrm{~mm}$. It is straightforward to readjust the design to cancel the end-field multipoles.

An alternative concept would be to use a single dipolelike design with laterally displaced poles (see Fig. 38) as discussed in Refs. [31-33].

\section{MUON STORAGE RING AND PERFORMANCE}

The storage ring in this study is assumed to be essentially identical to that in FS2. However, injection will be required in two opposite directions for the two differing signs. The injection lines must be designed such that when the train of one sign is traveling towards the detector, the train of the other sign is moving away from the detector. In this way the neutrinos of opposite kind arrive at well separated times, and the experiment can analyze their reactions separately. Another difference is that in this study, both straight sections must be designed with very high betas, so that the neutrino beams of both types are well collimated. Finally, it must be noted that the total energy deposited in the ring is doubled by the presence of equally intense muon beams, but now of two signs.

Losses are summarized in Table X. We define $\eta$ to be the probability that a muon makes it successfully into the storage ring. The number of decays $N_{\mu}$, of each sign, injected into the storage ring in a $10^{7}$ second year is given by

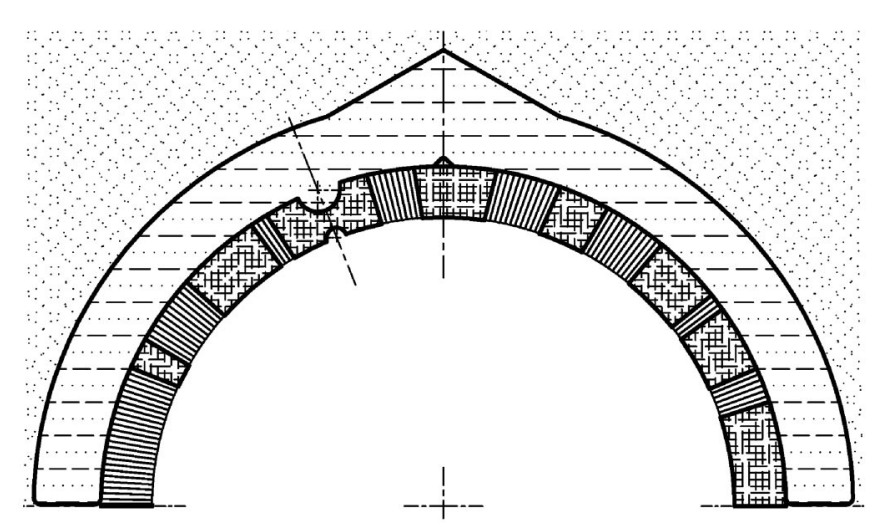

FIG. 38. Expanded view of the cross section of the superconducting combined-function magnet used in the $50 \mathrm{GeV}$ proton beam line for the J-PARC neutrino experiment [31].
TABLE X. Particle losses from cooling to storage ring.

\begin{tabular}{lc}
\hline \hline & Loss $\%$ \\
\hline Match into linac & 15 \\
Linac & 6.0 \\
RLA & 6.1 \\
FFAG 1 & 5.1 \\
FFAG 2 & 6.5 \\
Total losses & 33 \\
\hline \hline
\end{tabular}

$$
\begin{aligned}
N_{\mu} & =10^{7} f N_{p} \mu / p \eta \\
& \approx 10^{7} \times 15 \times\left(17 \times 10^{12}\right) \times 0.17 \times 0.67 \\
& \approx 2.9 \times 10^{20} .
\end{aligned}
$$

We define $\eta_{\text {straight }}$ to be the length of the straight section pointing to the detector divided by the circumference of the storage ring. The number of decays $N_{\nu}$, of both signs, in the storage ring, decaying towards the detector, in a $10^{7}$ second year is given by

$$
\begin{aligned}
N_{\nu} & =2 N_{\mu} \eta_{\text {straight }} \approx 2 \times 2.9 \times 10^{20} \times 0.35 \\
& \approx 2.0 \times 10^{20} .
\end{aligned}
$$

This is a factor of 2 greater than that reported in FS2. Note that if the proton driver power could be raised to $5 \mathrm{MW}$ (4 MW has been discussed in a further upgrade of the BNL AGS), then the number of neutrinos per year would match the high performance goal $\left(10^{21}\right)$ suggested at the first NuFact Workshop in Lyon, France [34].

\section{REQUIRED RESEARCH AND DEVELOPMENT}

As should be clear from the design descriptions, the muon-based neutrino factory is a demanding project. The machine makes use of novel components and techniques that are, in some cases, at or beyond the state of the art. For this reason, it is critical that research and development efforts to study these matters be carried out.

Each of the major systems has significant issues that must be addressed by research and development activities [5]. Component specifications need to be verified. For example, the cooling channel assumes a normal conducting rf cavity gradient of $15 \mathrm{MV} / \mathrm{m}$ at $201.25 \mathrm{MHz}$ in substantial magnetic fields. Observations of breakdown in $805 \mathrm{MHz}$ cavities have shown [35] serious reductions of attained rf gradients when the cavity is operated in a field. It is not clear how to scale these observations to the 201.25 MHz case, so experimental tests are urgently needed. If the required gradients cannot be achieved in the specified magnetic fields, then significant redesign will be needed.

The acceleration section demands high gradients from superconducting rf cavities at this frequency; our require- 
ments are somewhat beyond the performance reached to date for cavities in this frequency range [36].

Development and testing of efficient high-power $\mathrm{rf}$ sources at a frequency near $200 \mathrm{MHz}$ is also needed.

The ability of the target to withstand a proton beam power at $1 \mathrm{MW}$ and above must be confirmed.

Finally, an ionization cooling experiment should be undertaken to validate the implementation and performance of the cooling channel, and to confirm that our simulations of the cooling process are accurate.

\section{COST ESTIMATE: ASSUMPTIONS AND ALGORITHM}

For this study (ST2B) substantial effort has been directed at simplifying the design and thus hopefully reducing the costs of three major components of a neutrino factory: phase rotation, cooling, and the higher energy part of the muon acceleration. A preliminary comparison with FS2, which contained detailed cost estimates with significant engineering input, shows that a great deal of progress has been achieved. Starting from the FS2 work breakdown schedule, we derived element costs per unit length, integral rf voltage, or net acceleration. For all but the final FFAG acceleration, these costs were then applied to the ST2B parameters after scaling for magnetic fields, radii, stored energy, rf gradient, etc., For the FFAG costs a new cost algorithm had to be developed [27]. Further details on the costing algorithms and their application to this new design can be found in a recent reference [37].

There are a number of reasons why we believe this new design should be significantly less expensive than the previous one described in FS2. For phase rotation we have replaced $260 \mathrm{~m}$ of expensive induction linacs with $54 \mathrm{~m}$ of more conventional $\mathrm{rf}$ cavities. The $108 \mathrm{~m}$ long cooling channel in FS2 was replaced with an $80 \mathrm{~m}$ channel. The new cooling channel uses a simplified magnetic lattice [38] with reduced peak solenoid fields; it also replaces liquid hydrogen absorbers with solid $\mathrm{LiH}$ absorbers. For acceleration to $20 \mathrm{GeV}$ we have made use of recent advances in the development of nonscaling FFAG [39] accelerators to replace most of the recirculating linear accelerator used in FS2. There are of course some changes in the new design, such as the adopted increase in transverse acceptance in the accelerators, which will increase the costs over FS2. However, our examination shows that the design changes should lead to an overall reduction in costs.

A summary of the preliminary estimates for the percentage cost reductions for the ST2B neutrino factory design is presented in Table XI. It is most likely that a proton driver will first be built in conjunction with a neutrino superbeam experiment, so we begin the neutrino factory systems with the target and capture section. Excluding the proton driver
TABLE XI. Preliminary cost comparison between FS2 and ST2B.

\begin{tabular}{lc}
\hline \hline System & Reduced cost $\%$ \\
\hline Target, capture, 18 m drift & 98 \\
Bunching and phase rotation & 39 \\
Cooling & 60 \\
Acceleration & 77 \\
Ring & 100 \\
Total without driver and controls & 65 \\
\hline \hline
\end{tabular}

the new design should cost $\approx 35 \%$ less than the FS2 design.

\section{SUMMARY}

A new type of facility has been proposed that could have a tremendous impact on future neutrino experiments - the neutrino factory. In contrast to conventional muon neutrino beams, the neutrino factory would provide a source of electron neutrinos $\left(\nu_{e}\right)$ and antineutrinos $\left(\bar{\nu}_{e}\right)$ with very low systematic uncertainties on the beam fluxes and spectra. The experimental signature for $\nu_{e} \rightarrow \nu_{\mu}$ transitions is extremely clean, with very low background rates. Hence, Neutrino Factories would enable very sensitive oscillation measurements to be made.

A substantial neutrino factory research and development effort has been ongoing in the U.S. and elsewhere over the last few years, and significant progress has been made towards optimizing the design, developing and testing the required accelerator components, and significantly reducing the cost.

The novel facility described here represents a significant improvement over previous designs. New ideas in bunching, phase rotation, and ionization cooling have been incorporated into the design of the front end, which now captures both muon signs simultaneously. The nonscaling FFAG acceleration concept has been further developed and used for accelerating the muons up to the $20 \mathrm{GeV}$ design energy. The performance of the new system equals that of the earlier FS2, for each of two neutrino states $(\nu$ and $\bar{\nu})$ that are generated essentially simultaneously. The performance is thus effectively twice that of FS2. At the same time, the facility is simpler than that in FS2 and of the order of $35 \%$ less costly.

Research and development is also continuing to confirm needed component performance and establish the physical concepts used. Continued optimization is ongoing, and is expected to further improve performance and reduce the cost.

\section{ACKNOWLEDGMENTS}

We would like to thank C. Johnstone, S. Geer, and Y. Fukui for helpful discussions. This research was supported by the U.S. Department of Energy under Contract Nos. 
DE-AC02-98CH10886, DE-AC02-76CH03000, and DEAC03-76SF00098.

[1] Technical Report No. BNL-52623, edited by S. Ozaki, R. Palmer, M. Zisman, and J. Gallardo, 2001, http:// www.cap.bnl.gov/mumu/studyii/FS2-report.html.

[2] S. Geer, Phys. Rev. D 57, 6989 (1998); 59, 039903(E) (1999).

[3] M. M. Alsharóa et al., Phys. Rev. ST Accel. Beams 6, 081001 (2003).

[4] A. Blondel et al., Report No. CERN 2004-002 ECFA/ CERN.

[5] "APS Multi-Divisional Study of the Physics of Neutrinos," http://www.interactions.org/neutrinostudy/, sponsored by the American Physical Society Divisions of Nuclear Physics, Particles and Fields, Astrophysics, Physics of Beams, 2004.

[6] The Neutrino Factory and Muon Collider Collaboration Web page, http://www.cap.bnl.gov/mumu/.

[7] http://hepunx.rl.ac.uk/neutrino-factory/.

[8] http://muonstoragerings.web.cern.ch/muonstoragerings/.

[9] http://www-prism.kek.jp/nufactj/index.html.

[10] Fermilab Technical Report No. Fermilab-Pub-00/ 108-E, 2000, edited by N. Holtkamp and D. Finley, http: //www.fnal.gov/projects/muon_collider/nu-factory/ nu-factory.html.

[11] D. Neuffer, Report No. MUC-NOTE-269, 2003; also see D. Neuffer, Report No. MUC-NOTE-181, 2000. All MUC-NOTE papers are available from http://www-mucool.fnal.gov/notes/noteSelMin.html.

[12] D. Neuffer, Report No. MUC-NOTE-266, 2003.

[13] D. Neuffer and A. Van Ginneken, in Proceedings of the Particle Accelerator Conference, Chicago, 2001 (IEEE, Piscataway, NJ, 2001), http://accelconf.web.cern.ch/ AccelConf/p01/PAPERS/TPPH162.pdf

[14] A. Van Gineeken, Fermilab Technical Report No. MUCNOTE-220, 2001.

[15] R. Fernow, in Proceedings of the Particle Accelerator Conference, New York, 1999, edited by A. Luccio and W. MacKay (IEEE, Piscataway, NJ, 1999), p. 3020, latest version available at http://pubweb.bnl.gov/people/fernow/ icool/readme.html.

[16] N. Mokhov, in Proceedings of the Particle Accelerator Conference, Chicago, 2001 (Ref. [13]), http://accelconf.web.cern.ch/AccelConf/p01/PAPERS/FOAC010.pdf; see also http://www-ap.fnal.gov/MARS/.

[17] K. McDonald, in Proceedings of the Particle Accelerator Conference, Chicago, 2001 (Ref. [13]), p. 1583; H. G. Kirk et al., in Proceedings of the Particle Accelerator Conference, Chicago, 2001 (Ref. [13]), p. 1535; Ref. [1], Chap. 3. All Particle Accelerator Conference papers can be obtained from http://accelconf.web.cern.ch/ accelconf/.
[18] D. Neuffer, in Proceedings of the APS Study Workshop, ANL, 2004; http://www.cap.bnl.gov/mumu/study2a/notes/ neuffer.pdf

[19] Handbook of Accelerator Physics and Engineering, edited by A. Chao and M. Tigner (World Scientific, Singapore, 1999), p. 133.

[20] R. Fernow, Report No. MUC-NOTE-317, 2005.

[21] K. R. Symon, D. W. Kerst, L. W. Jones, L. J. Laslett, and K. M. Terwilliger, Phys. Rev. 103, 1837 (1956).

[22] C. Johnstone, W. Wan, and A. Garren, in Proceedings of the Particle Accelerator Conference, New York, 1999 (Ref. [15]), p. 3068.

[23] F. E. Mills and C. Johnstone, in Proceedings of the 4th International Conference on Physics Potential \& Development of $\mu^{+} \mu^{-}$Colliders, San Francisco, CA (UCLA, Los Angeles, CA, 1999), pp. 693-698.

[24] M. Ono et al., in Proceedings of the 9th Workshop on rf Superconductivity, Los Alamos, NM, 2000 (Los Alamos National Laboratory Report No. LA-13782-C).

[25] J. S. Berg, C. Johnstone, and D. Summers, in Proceedings of the Particle Accelerator Conference, Chicago, 2001 (Ref. [13]), p. 3323; D. J. Summers, hep-ex/0208010.

[26] J.S. Berg, Report No. MUC-CONF-ACCELERATION309, 2004, http://www-mucool.fnal.gov/notes/ noteSelMin.html

[27] R. B. Palmer and J.S. Berg, in Proceedings of the European Particle Accelerator Conference, Lucerne, Switzerland (CERN, Geneva, 2004), p. 902.

[28] Vector Fields Inc., computer program OPERA-3D.

[29] S. Koscielniak and C. Johnstone, in Proceedings of the 2003 Particle Accelerator Conference, edited by J. Chew, P. Lucas, and S. Webber (IEEE, Piscataway, NJ, 2003), pp. 1831.

[30] S. Caspi and R. Hafalia, LBNL Report No. SC-MAG-839, 2004.

[31] T. Nakamoto et al., IEEE Trans. Appl. Supercond. 14, 616 (2004).

[32] T. Ogitsu et al., IEEE Trans. Appl. Supercond. 14, 604 (2004).

[33] T. Nakamoto et al., in Proceedings of the Particle Accelerator Conference, Knoxville, TN, 2005 (IEEE, Piscataway, NJ, 2005), p. 495.

[34] Proceedings of the ICFA/ECFA Workshop NUFACT'99: Neutrino Factories based on Muon Storage Rings, Lyon France, edited by N. Autin [Nucl. Instrum. Methods Phys. Res., Sect. A 451, 1 (2000)].

[35] J. Norem, et al., Phys. Rev. ST Accel. Beams 6, 072001 (2003); Report No. MUC-NOTE-226, 2001.

[36] R. L. Geng et al., in [29], pp. 1309-1311.

[37] R. Palmer and M. Zisman, Report No. MUC-NOTE-322, 2005.

[38] M. A. Green, R. Byrns, and S. J. St. Lorant, Adv. Cryog. Eng. 37, 637 (1991); see also K. Hagiwara et al. (Particle Data Group), Phys. Rev. D 66, 010001 (2002).

[39] J.S. Berg, R. Fernow, and R. B. Palmer, in Proceedings of the FFAG Workshop, Vancouver, Canada, 2004, http:// www.triumf.ca/ffag2004/ 